\title{
Dark matter assisted lepton anomalous magnetic moments and neutrino masses
}

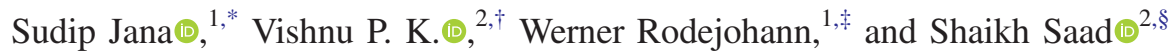 \\ ${ }^{1}$ Max-Planck-Institut für Kernphysik, Saupfercheckweg 1, 69117 Heidelberg, Germany \\ ${ }^{2}$ Department of Physics, Oklahoma State University, Stillwater, Oklahoma 74078, USA
}

(Received 11 August 2020; accepted 22 September 2020; published 8 October 2020)

\begin{abstract}
We propose a framework that addresses the origin of neutrino mass, explains the observed discrepancies in the electron and the muon anomalous magnetic moments (AMMs) data, and incorporates the dark matter (DM) relic abundance. Both the neutrino mass and the lepton AMMs are generated at one-loop level mediated by a common set of beyond the Standard Model (SM) states. In this class of models, the SM is extended with vectorlike charged fermion and scalar multiplets, all odd under an imposed $\mathcal{Z}_{2}$ symmetry, which stabilizes the fermionic or scalar DM candidate residing in one of them. Two scalar multiplets appear in the AMM loops, thus allowing for different signs of their contributions, in agreement with the observed discrepancies which are of opposite sign for electron and muon. The vectorlike fermions give rise to large new physics contributions to the lepton AMMs via chirally enhanced terms that are proportional to their mass. To demonstrate the viability of this framework, we perform a detailed study of a particular model for which a fit to the neutrino masses and mixing together with lepton AMMs are provided. Furthermore, DM phenomenology and collider signatures are explored.
\end{abstract}

DOI: 10.1103/PhysRevD.102.075003

\section{INTRODUCTION}

The origin of the neutrino mass is among the most crucial problems of the Standard Model (SM) of particle physics. On the other hand, the almost century old dark matter (DM) problem is another tremendous puzzle yet to be solved. The most straightforward approach to this issue is the particle nature of the DM (for a review, see Ref. [1]). One of the many popular mechanisms for neutrino mass is the radiative one (for a recent review, see Ref. [2,3]) due to the natural accessibility of the involved particles at colliders and low energy experiments.

There have been lots of attempts in the literature to combine these two seemingly uncorrelated issues, one of the most prominent example being the scotogenic model [4]. In such models, the particles mediating the loop(s) that generate neutrino mass are dark matter. Typically, new symmetries beyond the SM are required to stabilize the DM and in some cases to forbid the tree-level neutrino mass

\footnotetext{
*sudip.jana@mpi-hd.mpg.de

†vipadma@okstate.edu

${ }^{*}$ werner.rodejohann@mpi-hd.mpg.de

§shaikh.saad@okstate.edu
}

Published by the American Physical Society under the terms of the Creative Commons Attribution 4.0 International license. Further distribution of this work must maintain attribution to the author(s) and the published article's title, journal citation, and DOI. Funded by SCOAP ${ }^{3}$. contributions; for systematic studies along this line, see for example Refs. [5-10]. The details of these models largely depend on the nature of the imposed symmetries and the needed particle content. However, common features of these models are that (i) neutrino mass is generated via quantum corrections at a given loop order; (ii) DM candidates naturally arise due to symmetry reasons; and (iii) owing to the loop suppression, the new physics (NP) scale can be around the $\mathrm{TeV}$ scale without making the Yukawa couplings unnecessarily small, which provides a way to test these models at low energies.

Aside from neutrino mass and DM, there has been a longstanding tension between the SM prediction [11-14] and the experimental measured value [15] of the muon anomalous magnetic moment (AMM). Additionally, the recently measured fine-structure constant $\alpha$ using Caesium atoms with unprecedented precision [16] implies a deviation of the electron AMM from the SM value [17] of opposite sign compared to the muon AMM. The experimental measurements point toward about $2.5 \sigma$ and $3.7 \sigma$ tensions for the electron and the muon AMMs, respectively. More precisely, the corresponding discrepancies are given as

$$
\begin{aligned}
& \Delta a_{e}=a_{e}^{\exp }-a_{e}^{\mathrm{SM}}=-(8.7 \pm 3.6) \times 10^{-13}, \\
& \Delta a_{\mu}=a_{\mu}^{\exp }-a_{\mu}^{\mathrm{SM}}=(2.79 \pm 0.76) \times 10^{-9} .
\end{aligned}
$$

Since the AMMs of light charged leptons $\left(a_{\ell}=(g-2)_{\ell} / 2\right.$, $\ell=e, \mu)$ are measured with excellent accuracy in the 
experiments, and their corresponding theory values are computed with outstanding precision, these observed tensions strongly point toward physics beyond the SM. Therefore, these results recently have entertained a lot of interest in the particle physics community; for attempts to simultaneously explain these discrepancies, see Refs. [18-45]. For previous analyses of nonsupersymmetric models that accommodate only DM and $(g-2)_{\mu}$, see Refs. [46-54], and for studies that make a connection between radiative neutrino mass generation and $(g-2)_{\mu}$, see Refs. [55-62].

To address both $(g-2)_{e}$ and $(g-2)_{\mu}$, NP may appear at low scale; see for example Ref. [36]. Models of these types are highly constrained from beam dump experiments, Belle and $B A B A R$, which may eventually rule out such scenarios in the near future. We, on the other hand, are interested in scenarios where NP emerges at heavy scale. ${ }^{1}$ To incorporate large deviations for $\Delta a_{\ell}$ given in Eqs. (1.1) and (1.2) from heavy NP, a chirality flip of a heavy state must take place inside the loop. This can be achieved with TeV scale scalar leptoquarks [35,44] or vectorlike fermions [20]. These studies, however, made no connection with either neutrino mass or DM issues.

In this work, we bring the issues of the origin of neutrino mass, the DM problem, and the electron and muon AMM puzzles under the same umbrella and propose a framework for their explanations in a minimalistic approach. In our proposed setup, the particle content of the SM is extended by three generations of vectorlike fermions and three scalar multiplets. Furthermore, the model is supplemented with a $\mathcal{Z}_{2}$ symmetry, under which only the beyond the Standard Model (BSM) particles are assumed to be odd. Via the propagation of these BSM multiplets, neutrino mass generation as well as new physics contributions to the lepton anomalous magnetic moments of the correct order appear at one-loop level. Two scalar multiplets, and thus two sets of Yukawa couplings, appear in the AMM loops, thus allowing for different signs of their contributions, in agreement with the observed discrepancies which are of opposite sign for electron and muon. The lightest of the neutral BSM particles is stabilized by the imposed $\mathcal{Z}_{2}$ symmetry, which serves as the DM candidate.

The paper is built as follows. In Sec. II, we address which model classes may solve the AMM discrepancies and at the same time generate neutrino mass radiatively with the same set of new multiplets. From the list of models, in Sec. III, we perform a detailed analysis of one of them, analyzing the scalar sector, performing a fit to the AMM and neutrino mass observables, discussing the dark matter

\footnotetext{
${ }^{1}$ Both heavy and light new physics are not expected to influence the MUonE experiment [63], which will directly measure the crucial hadronic vacuum polarization contribution to the muon AMM; see Refs. [64,65].
}

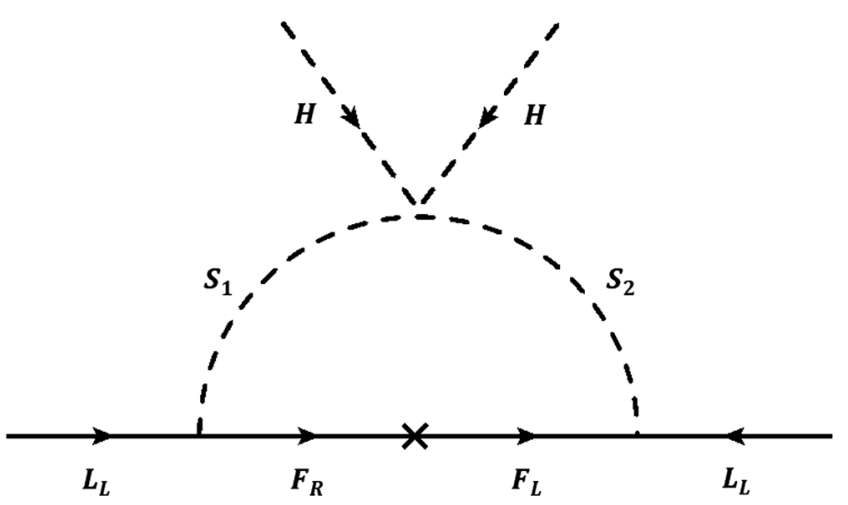

FIG. 1. Representative Feynman diagram for generating neutrino mass.

phenomenology, and outlining collider phenomenology. We conclude in Sec. IV.

\section{FRAMEWORK}

Due to its simplicity, we start our discussion with the scotogenic model [4], which employs three generations of singlet Majorana fermions ${ }^{2} N(1,1,0)$ and an inert Higgs doublet $\phi(1,2,1 / 2)$, both odd under an imposed $\mathcal{Z}_{2}$ symmetry. The neutrino mass is generated at the oneloop level via the generic diagram shown in Fig. 1, with $S_{1}=S_{2} \equiv \phi$ and $F_{L}=F_{R} \equiv N \quad$ (Majorana fermion). However, a combined explanation of lepton AMMs along with reproducing realistic neutrino masses and mixings cannot be accommodated, since the proportionality relation $\left|a_{\mu}\right| \propto m_{\mu}^{2}$ requires large Yukawa couplings, which would generate too large rates for charged lepton flavor violating (cLVF) processes like $\mu \rightarrow e \gamma$. Quantifying this tension very roughly is possible as follows. In the scotogenic model, neutrino mass is given by (assuming that all new particle masses are of order $\mathrm{TeV}$ )

$\mathcal{M}_{\nu} \sim \frac{\lambda\langle H\rangle^{2}}{32 \pi^{2}} \frac{y^{2}}{M_{N}} \sim 0.05\left(\frac{\mathrm{TeV}}{M_{N}}\right)\left(\frac{y}{2.3 \times 10^{-5}}\right)^{2} \mathrm{eV} ;$

here, $\langle H\rangle$ is the vacuum expectation value (VEV) of the SM Higgs doublet, and $\lambda$ represents the quartic coupling in the scalar potential that is responsible for breaking the degeneracy between the real and the imaginary parts of the neutral component residing in the inert doublet, which we have taken to be unity in the second line. In the above formula, flavor indices are ignored, and $y$ is the Yukawa coupling of the singlet fermion $N$ with lepton doublets and the inert scalar doublet. This shows that to get the correct order of neutrino mass $\left(m_{\nu} \sim 0.05 \mathrm{eV}\right)$ one requires $y \sim \mathcal{O}\left(10^{-5}\right)$. On the other hand, the contribution to the magnetic moment is

\footnotetext{
${ }^{2}$ Our convention is $Q=I_{3}+Y$.
} 
$-\Delta a_{\mu} \sim m_{\mu}^{2} \frac{|y|^{2}}{32 \pi^{2} M_{S}^{2}} \sim 10^{-21}\left(\frac{M_{S}}{\mathrm{TeV}}\right)^{-2}\left(\frac{y}{10^{-5}}\right)^{2}$.

Apart from the fact that the sign of $\Delta a_{\mu}$ is actually not correct in the scotogenic model, these estimates show that a simultaneous explanation of neutrino mass and the anomalous magnetic moment is not possible. Moreover, the branching ratio for $\mu \rightarrow e \gamma$ provides additional constraints, namely,

$\operatorname{BR}(\mu \rightarrow e \gamma) \sim \frac{3 \alpha y^{4}}{32 \pi G_{F}^{2} M_{S}^{4}} \sim 10^{-26}\left(\frac{M_{S}}{\mathrm{TeV}}\right)^{-4}\left(\frac{y}{10^{-5}}\right)^{4}$,

with $S$ a scalar particle of the model. Too large rates would appear for order 1 Yukawas. A detailed parameter scan confirms such statements [66,67].

The sign of the muon AMM could be changed by a minimal addition of one more scalar, which provides a freedom to choose the sign of the product of the Yukawa coupling in the AMM contributions. This would utilize either of the two one-loop diagrams presented in Fig. 2. For the scotogenic model, both these diagrams are identical, and the loop can be completed by introducing a singly charged scalar, that is, $S_{3}=S_{4} \equiv \eta(1,1,1)$. However, the smallness of the implied AMM contribution remains and can be quantified as follows. The presence of $\eta^{+}$, with different hypercharge than the inert doublet and Yukawa coupling $y^{\prime}$, allows both left-handed and right-handed charged leptons in the external legs (unlike the scotogenic model that involves only left-handed charged fermions) and provides enhanced contribution to lepton AMM that is proportional to the mass of $N_{R}$. Then, the formula given in Eq. (2.2) has the following modified form,

$-\Delta a_{\mu} \sim \frac{m_{\mu}}{8 \pi^{2} M_{S}^{2}} y y^{\prime} \theta M_{N} \sim 10^{-12}\left(\frac{M_{\mathrm{NP}}}{\mathrm{TeV}}\right)^{-1}\left(\frac{y}{10^{-5}}\right) y^{\prime} \theta$,

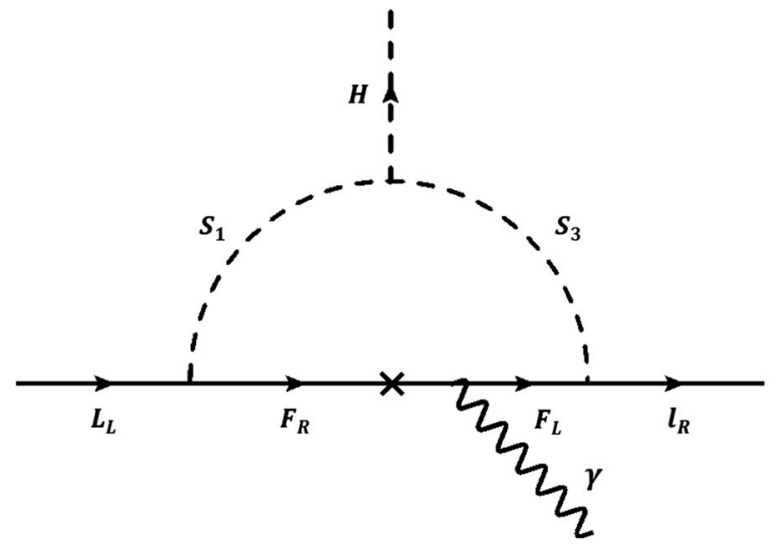

where $\theta \leq 1$ represents the mixing angle between the two singly charged states, $M_{\mathrm{NP}}$ is common new physics scale of the new particles, and in the second line we have assume the dominance of one of the terms toward lepton AMM to maximize the effect. In this scenario, the neutrino mass is determined by the same Yukawa coupling $y$ as before; however, both $y$ and $y^{\prime}$ play a role in lepton AMM. This implies that to maximize lepton AMM $y^{\prime}$ needs to be chosen as large as possible, which does not affect the neutrino mass. However, even with $y^{\prime} \sim \mathcal{O}(1)$, much higher values than $y \sim \mathcal{O}\left(10^{-5}\right)$ are required to explain the AMM, which would be in conflict with neutrino mass and cLFV for $\mathrm{TeV}$ scale new particles. For further clarity, we remind the readers that nontrivial flavor structure of $y$ is required to accommodate neutrino mixings; on the other hand, $y^{\prime}$ can be simply taken to be diagonal and hence would not lead to cLFV, as will be discussed shortly. Such correlations can be avoided if the Yukawa coupling $y$ does not participate in explaining $(g-2)_{\ell}$. This is precisely what we try to achieve in an economical fashion within our framework.

The same conclusion can be reached for any similar model with Majorana fermions running in the loop in Fig. 1 that transform nontrivially under the $S U(2)_{L}$ group, for example $F \sim(1,3,0)$ (for this choice, one again gets $\left.S_{1}=S_{2} \equiv \phi\right)$.

The above arguments are also changed if hypercharged vectorlike Dirac fermions instead of Majorana fermions are introduced. This requirement still allows the Dirac fermions to have a bare mass term (vectorlike under the SM) and simultaneously demands that $S_{1} \neq S_{2}$ in Fig. 1, owing to the new fermions carrying $Y \neq 0$. Consequently, two different Yukawa coupling matrices play a role in generating neutrino mass, which resolves the above-mentioned issues. With only these two scalars present in a theory, a mass flip of the vectorlike fermion cannot be realized for lepton AMM contributions; hence, a third scalar of either $S_{3}$ or $S_{4}$ must be introduced for such a purpose as shown in Fig. 2. The presence of at least three different Yukawa

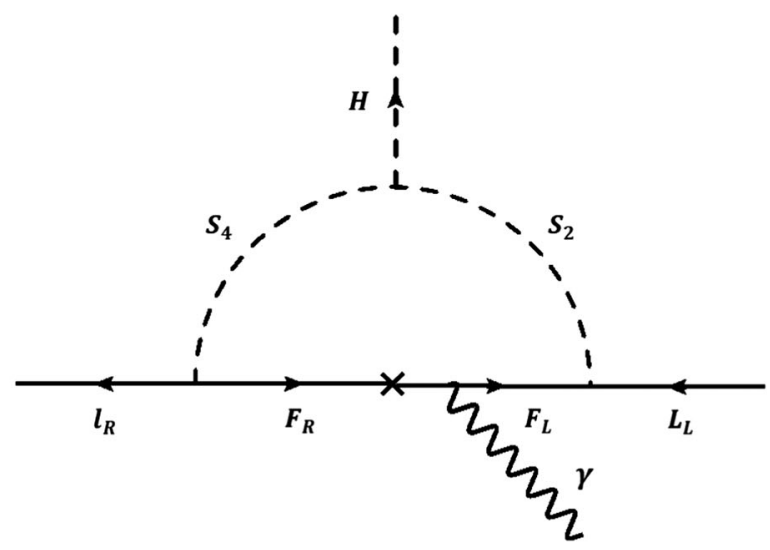

FIG. 2. New physics contributions to $(g-2)_{\ell}$. The outgoing photon can be emitted from the internal fermion or boson line (or both) depending on the model. 
TABLE I. Here, we have listed only the viable models up to $S U(2)_{L}$ triplets that satisfy our required criteria; see text for details. By following our method, models involving higher-dimensional representations can be constructed trivially. Multiplets containing a potential dark matter candidate are shown in red.

\begin{tabular}{|c|c|c|c|c|c|c|c|}
\hline Multiplets & Model I & Model II & Model III & Model IV & Model V & Model VI & Model VII \\
\hline$F_{L, R}$ & $(1,1,-1)$ & $(1,1,-1)$ & $(1,2,-1 / 2)$ & $(1,2,-1 / 2)$ & $(1,2,-3 / 2)$ & $(1,3,1)$ & $(1,3,1)$ \\
\hline$S_{1}$ & $(1,2,1 / 2)$ & $(1,2,1 / 2)$ & $(1,1,0)$ & $(1,1,0)$ & $(1,1,1)$ & $(1,2,3 / 2)$ & $(1,2,3 / 2)$ \\
\hline$S_{2}$ & $(1,2,3 / 2)$ & $(1,2,3 / 2)$ & $(1,3,1)$ & $(1,3,1)$ & $(1,3,-2)$ & $(1,2,1 / 2)$ & $(1,2,1 / 2)$ \\
\hline$S_{3}$ & $(1,1,0)$ & $\ldots$ & $(1,2,1 / 2)$ & & $(1,2,1 / 2)$ & $(1,3,2)$ & \\
\hline$S_{4}$ & $\ldots$ & $(1,1,2)$ & $\ldots$ & $(1,2,3 / 2)$ & $\ldots$ & $\ldots$ & $(1,3,0)$ \\
\hline
\end{tabular}

couplings allows one to disentangle contributions to AMM, neutrino mass, and cLFV and, as mentioned above, to control the sign of the AMM contributions. As aforementioned, in our setup, all the BSM multiplets are assumed to be odd under $\mathcal{Z}_{2}$; consequently, the lightest among the neutral component fields can play the role of DM and successfully explain the DM relic abundance. It is to be pointed out that the requirement of the new fermions carrying nonzero hypercharge is an outcome of the DMstabilizing $\mathcal{Z}_{2}$ symmetry, if the diagrams in Figs. 1 and 2 are supposed to exist. This could be relaxed if a different discrete or continuous symmetry is chosen to build a model, which we do not pursue. In this work, we strictly stick to $\mathcal{Z}_{2}$ symmetry for the fixed topology as in Fig. 1 to generate neutrino mass. For general analyses of various topologies of neutrino mass arising by utilizing exotic vectorlike fermions, see for example Refs. [68-70].

From these Feynman diagrams and the above discussion, one sees that a common set of multiplets, either $\left\{F_{L, R}, S_{1}\right\}$ or $\left\{F_{L, R}, S_{2}\right\}$ depending on the model, plays role in both the neutrino mass generation and in accommodating lepton AMMs data. With $\mathrm{TeV}$ scale vectorlike fermions, the appropriate scale of neutrino masses can be naturally reproduced with Yukawa couplings that are comparable in order with the SM charged fermion Yukawa couplings. Furthermore, even with $\mathrm{TeV}$ scale vectorlike fermions, the required large contributions toward both $(g-2)_{e}$ and $(g-2)_{\mu}$ can be promptly obtained via chirality enhancement.

Having stated our criteria, the exercise is now to find a set of vectorlike fermions and scalars that allow for the topologies in Figs. 1 and 2. This leads to the models summarized in Table I. Here, we have listed only the viable models up to $S U(2)_{L}$ triplets that satisfy our abovementioned criteria. Multiplets that contain a neutral component and thus a potential DM candidate are shown in red. By following our methodology, models involving higher-dimensional representations can be constructed trivially. It is beyond the scope of this work to study each of these models in detail. Instead, in the next section we perform a detailed analysis of the first model (model I) in the list.

\section{DETAILS OF MODEL I}

In this section, we perform a detailed analysis of model I. In this model, the SM particle content is extended by three singly charged vectorlike fermions $F_{L, R}$ and three scalars: a singlet and two doublets under the $S U(2)_{L}$ group. One of the doublets has hypercharge $1 / 2$, and the other has $3 / 2$. As already mentioned, under the imposed $\mathcal{Z}_{2}$ symmetry, the SM particles are even, whereas all the BSM states are odd. The full quantum numbers of the BSM multiplets are summarized in Table II.

With this particle content, the most general Yukawa Lagrangian consistent with all symmetries is given as

$$
\begin{aligned}
-\mathcal{L}_{Y}= & y_{H} \bar{L}_{L} \ell_{R} H+y_{1} \bar{L}_{L} F_{R} \phi_{1}+y_{2}{\overline{L^{c}}}_{L} F_{L} i \tau_{2} \phi_{2} \\
& +\frac{y_{3}}{\sqrt{2}} \bar{\ell}_{R} F_{L} \eta+M_{F} \bar{F}_{L} F_{R}+\text { H.c. }
\end{aligned}
$$

Here, $L_{L}$ is a left-handed lepton doublet, $\ell_{R}$ is a righthanded lepton, and $H$ is the SM Higgs doublet. In Eq. (3.1), for simplicity, we have suppressed generation indices. Yukawa couplings of the quarks remain unchanged compared to the SM; hence, we only focus on the leptonic sector. We work in the basis where the Yukawa coupling $y_{H}$ and the vectorlike fermion mass matrix $M_{F}$ are diagonal. The three new Yukawa couplings matrices $y_{1,2,3}$ are in general arbitrary.

\section{A. Scalar sector}

The scalar sector of the full model consists of three neutral $C P$-even states $h, S_{1,2}^{0}$; one neutral $C P$-odd $A^{0}$;

TABLE II. Quantum numbers of the BSM multiplets for Model I. Both $\phi_{1}$ and $\eta$ contain a DM candidate.

\begin{tabular}{lcc}
\hline \hline Multiplets & $S U(3)_{C} \times S U(2)_{L} \times U(1)_{Y}$ & $\mathcal{Z}_{2}$ \\
\hline Scalars & $\phi_{1}\left(1,2, \frac{1}{2}\right)$ & - \\
& $\phi_{2}\left(1,2, \frac{3}{2}\right)$ & - \\
& $\eta(1,1,0)$ & - \\
Vectorlike fermion & $F_{L, R}(1,1,-1)$ & - \\
\hline \hline
\end{tabular}


two singly charged $S_{1,2}^{+}$; and a doubly charged $S^{++}$. Here, $h$ is identified with the SM Higgs, which does not mix with the rest of the two states $S_{1,2}^{0}$ due to the imposed $\mathcal{Z}_{2}$ symmetry. The lightest between these two states $S_{1,2}^{0}$ is identified as the DM. Moreover, we assume the BSM multiplets $\eta$ and $\phi_{1}$ do not accrue any VEV; hence, the Goldstone bosons $G^{0}, G^{ \pm}$originate entirely from the SM Higgs doublet $H$. The complete scalar potential for model I is given as

$$
\begin{aligned}
V= & -\mu_{H}^{2} H^{\dagger} H+\sum_{\varphi}^{\left\{\phi_{1}, \phi_{2}\right\}} \mu_{\varphi}^{2} \varphi^{\dagger} \varphi+\mu_{\eta}^{2} \eta^{2} \\
& +\left(\mu_{5} H^{\dagger} \phi_{1} \eta+\text { H.c. }\right)+\sum_{\varphi}^{\left\{H, \phi_{1}, \phi_{2}\right\}} \lambda_{\varphi}\left(\varphi^{\dagger} \varphi\right)^{2}+\lambda_{\eta} \eta^{4} \\
& +\sum_{\varphi<\varphi^{\prime}}^{\left\{H, \phi_{1}, \phi_{2}\right\}} \lambda_{\varphi \varphi^{\prime}}\left(\varphi^{\dagger} \varphi\right)\left(\varphi^{\prime \dagger} \varphi^{\prime}\right)+\sum_{\varphi}^{\left\{H, \phi_{1}, \phi_{2}\right\}} \lambda_{\varphi \eta}\left(\varphi^{\dagger} \varphi\right) \eta^{2} \\
& +\sum_{\left.\varphi<, \phi_{1}, \phi_{2}\right\}} \lambda_{\varphi \varphi^{\prime}}^{\prime}\left(\varphi^{\dagger} \varphi^{\prime}\right)\left(\varphi^{\prime \dagger} \varphi\right)+\left\{\lambda_{H \phi_{1}}^{\prime \prime}\left(H^{\dagger} \phi_{1}\right)^{2}+\text { H.c. }\right\} \\
& +\left\{\lambda_{\phi_{1} \phi_{2}}^{\prime \prime}\left(H \epsilon \phi_{1}\right)\left(\phi_{2}^{\dagger} H\right)+\text { H.c. }\right\} .
\end{aligned}
$$

We now derive the masses of the physical Higgs particles from the above potential. The mass-squared matrix $\mathcal{M}_{S^{0}}^{2}$ for the two $C P$-even states, written in the $\left\{\eta^{0}, \operatorname{Re}\left(\phi_{1}^{0}\right)\right\}$ basis, is

$$
\mathcal{M}_{S^{0}}^{2}=\left(\begin{array}{cc}
2 \mu_{\eta}^{2}+\lambda_{H \eta} v_{H}^{2} & \mu_{5} v_{H} \\
\mu_{5} v_{H} & \mu_{\phi_{1}}^{2}+\frac{\left(\lambda_{H \phi_{1}}+\lambda_{H \phi_{1}}^{\prime}+2 \lambda_{H \phi_{1}}^{\prime \prime}\right)}{2} v_{H}^{2}
\end{array}\right) .
$$

The scalars that do not mix with any other fields are the SM Higgs, the $C P$-odd scalar, and the doubly charged scalar. The corresponding squared masses are

$$
\begin{gathered}
m_{h}^{2}=2 \lambda_{H} v_{H}^{2}, \\
m_{A^{0}}^{2}=\mu_{\phi_{1}}^{2}+\frac{\left(\lambda_{H \phi_{1}}+\lambda_{H \phi_{1}}^{\prime}-2 \lambda_{H \phi_{1}}^{\prime \prime}\right)}{2} v_{H}^{2}, \\
m_{S^{ \pm \pm}}^{2}=\mu_{\phi_{2}}^{2}+\frac{\lambda_{H \phi_{2}}}{2} v_{H}^{2},
\end{gathered}
$$

where $\phi_{A}$ is $\operatorname{Im}\left(\phi_{1}^{0}\right)$. Finally, the mass-squared matrix for the singly charged scalars in a basis of $\left(\phi_{1}^{ \pm}, \phi_{2}^{ \pm}\right)$reads

$\mathcal{M}_{S^{ \pm}}^{2}=\left(\begin{array}{cc}\mu_{\phi_{1}}^{2}+\frac{\lambda_{H \phi_{1}}}{2} v_{H}^{2} & -\frac{\lambda_{\phi_{1} \phi_{2}}^{\prime \prime}}{2} v_{H}^{2} \\ -\frac{\lambda_{\phi_{1} \phi_{2}}^{\prime \prime}}{2} v_{H}^{2} & \mu_{\phi_{2}}^{2}+\frac{\left(\lambda_{H \phi_{2}}+\lambda_{H \phi_{2}}^{\prime}\right)}{2} v_{H}^{2}\end{array}\right)$.
Moreover, the mixing angle $\alpha(\gamma)$ between the two mixed $C P$-even (singly charged) states can be calculated from

$$
\begin{aligned}
& \tan 2 \alpha=\frac{2 \mu_{5} v_{H}}{\left(\mathcal{M}_{S^{0}}^{2}\right)_{11}-\left(\mathcal{M}_{S^{0}}^{2}\right)_{22}}, \\
& \tan 2 \gamma=\frac{\lambda_{\phi_{1} \phi_{2}}^{\prime \prime} v_{H}^{2}}{\left(\mathcal{M}_{S^{ \pm}}^{2}\right)_{22}-\left(\mathcal{M}_{S^{ \pm}}^{2}\right)_{11}} .
\end{aligned}
$$

We note that the presence of nonzero $\alpha$ is crucial for generating the AMMs and for the dark matter phenomenology. Nonzero $\gamma$ is required to generate neutrino mass. Moreover, between the two neutral physical states $S_{1,2}^{0}$, we will assume $S_{1}^{0}$ to be the lighter one and identify it as the DM candidate. Its decomposition in terms of the original fields is given by $S_{1}^{0}=\eta^{0} \cos \alpha+\operatorname{Re}\left(\phi_{1}^{0}\right) \sin \alpha$.

\section{B. Lepton anomalous magnetic moments}

In the present setup, we assume the vectorlike fermions to reside around the $\mathrm{TeV}$ scale. In contrast to the scotogenic case, having such a heavy mass does not require large Yukawa couplings to incorporate the $\Delta a_{\ell}$ data given in Eqs. (1.1) and (1.2). Large enough corrections to the lepton AMMs naturally arise due to a chirality flip of the vectorlike fermions on the internal line, as can be seen from Fig. 2. Moreover, the sign difference for $\Delta a_{e}$ and $\Delta a_{\mu}$ is obtained by appropriately choosing the sign of the product of the Yukawa couplings that enter in this chirality enhanced AMM term. We derive the complete NP contributions toward $(g-2)_{\ell}$ that is given by [71]

$$
\begin{aligned}
\Delta a_{\ell}= & \frac{m_{\ell}}{16 \pi^{2}} \sum_{j=1}^{3}\left[\sum_{k=1}^{2} \operatorname{Re}\left(Y_{L, k}^{* \ell j} Y_{R, k}^{\ell j}\right) \frac{M_{F_{j}}}{M_{S_{k}}^{2}} G\left(\frac{M_{F_{j}}^{2}}{M_{S_{k}}^{2}}\right)\right. \\
& +\frac{m_{\ell}^{2}}{4 \pi^{2}} \sum_{k=1}^{3}\left(\left|Y_{L, k}^{\ell j}\right|^{2}+\left|Y_{R, k}^{\ell j}\right|^{2}\right) \frac{1}{M_{S_{k}}^{2}} \tilde{G}\left(\frac{M_{F_{j}}^{2}}{M_{S_{k}}^{2}}\right) \\
& \left.+\frac{m_{\ell}^{2}}{4 \pi^{2}}\left|Y_{L, 4}^{\ell j}\right|^{2} \frac{1}{M_{S_{4}}^{2}} \tilde{G}\left(\frac{M_{F_{j}}^{2}}{M_{S_{4}}^{2}}\right)\right],
\end{aligned}
$$

where we have defined $S_{1}=S_{1}^{0}, S_{2}=S_{2}^{0}, S_{3}=A^{0}$, and $S_{4}=S^{ \pm \pm}$. The expressions for the loop functions are

$$
\begin{gathered}
G(x)=\frac{3-4 x+x^{2}+2 \ln (x)}{(x-1)^{3}}, \\
\tilde{G}(x)=\frac{2+3 x-6 x^{2}+x^{3}+6 x \ln x}{24(1-x)^{4}} .
\end{gathered}
$$

The rescaled Yukawa couplings appearing in Eq. (3.9) are defined by

$$
Y_{L, 1}^{\ell j}=\frac{\sin \alpha}{\sqrt{2}}\left(y_{1}\right)_{\ell j}, \quad Y_{L, 2}^{\ell j}=\frac{\cos \alpha}{\sqrt{2}}\left(y_{1}\right)_{\ell j},
$$




$$
\begin{gathered}
Y_{R, 1}^{\ell j}=\frac{\cos \alpha}{\sqrt{2}}\left(y_{3}\right)_{\ell j}, \quad Y_{R, 2}^{\ell j}=\frac{-\sin \alpha}{\sqrt{2}}\left(y_{3}\right)_{\ell j}, \\
Y_{L, 3}^{\ell j}=i \frac{\sin \alpha}{\sqrt{2}}\left(y_{1}\right)_{\ell j}, \quad Y_{R, 3}^{\ell j}=0, \quad Y_{L_{\ell j}, 4}^{\ell, 4}=-\left(y_{2}\right)_{\ell j} .
\end{gathered}
$$

It should be pointed out that the very first term (chirality flip term) in Eq. (3.9) dominates; the remaining contributions can be safely ignored for our case, which we have confirmed numerically. In our setup, the contribution from the SM Higgs $h$ remains unchanged, which is already part of $a_{\ell}^{\mathrm{SM}}$. We stress here that for $\alpha=0$ the dominating first contribution to the AMMs would vanish. This can be understood from the expressions in (3.2) and (3.8). Vanishing $\alpha$ would correspond to vanishing $\mu_{5}$ and thus no triple-scalar coupling of $\phi_{1}$ with $\eta$ and the SM Higgs. This in turn would correspond to the absence of the AMM diagram in Fig. 2.

The off-diagonal elements in the Yukawa couplings $y_{1,3}$ will lead to cLFV processes such as $\ell \rightarrow \ell^{\prime} \gamma$. Due to the same chirality enhancement effects via the vectorlike fermions, these processes impose severe constraints on these off-diagonal Yukawa couplings. Amplitudes of these cLFV processes can be straightforwardly computed for our scenario; however, for the simplicity of our work, we assume the two Yukawa coupling matrices $y_{1,3}$ to be diagonal (meaning, small off-diagonal entries are omitted for our analysis). However, nonzero but small off-diagonal entries have no impact on the results obtained in this work. There are also very stringent constraints that arise from the lepton dipole moments (for a review, see Ref. [72]) measurements for complex couplings. We avoid these constraints by demanding these $y_{1,3}$ couplings to be real. For completeness, here we present the generic expressions for the cLFV process $\ell \rightarrow \ell^{\prime} \gamma$ for our model,

$$
\begin{aligned}
\operatorname{BR}(\ell \rightarrow & \left.\ell^{\prime} \gamma\right)=\frac{m_{\ell}^{3} \tau_{\ell} \alpha}{4096 \pi^{4}}\left(\left|A^{\ell \ell^{\prime}}\right|^{2}+\left|A^{\ell^{\prime} \ell}\right|^{2}\right), \\
A^{\ell \ell^{\prime}}= & \sum_{j=1}^{3}\left[\sum_{k=1}^{2} Y_{L, k}^{* \ell^{\prime} j} Y_{R, k}^{\ell j} \frac{M_{F_{j}}}{M_{S_{k}}^{2}} G\left(\frac{M_{F_{j}}^{2}}{M_{S_{k}}^{2}}\right)\right. \\
& \left.-\sum_{k=1}^{4} Y_{L, k}^{* \ell^{\prime} j} Y_{L, k}^{\ell j} \frac{4 m_{\ell}}{M_{S_{k}}^{2}} \tilde{G}\left(\frac{M_{F_{j}}^{2}}{M_{S_{k}}^{2}}\right)\right], \\
A^{\ell^{\prime} \ell}= & \sum_{j=1}^{3}\left[\sum_{k=1}^{2} Y_{L, k}^{* \ell j} Y_{R, k}^{\ell^{\prime} j} \frac{M_{F_{j}}}{M_{S_{k}}^{2}} G\left(\frac{M_{F_{j}}^{2}}{M_{S_{k}}^{2}}\right)\right. \\
& \left.-\sum_{k=1}^{4} Y_{R, k}^{* \ell j} Y_{R, k}^{\ell^{\prime} j} \frac{4 m_{\ell}}{M_{S_{k}}^{2}} \tilde{G}\left(\frac{M_{F_{j}}^{2}}{M_{S_{k}}^{2}}\right)\right] ;
\end{aligned}
$$

here, $\tau_{\ell}$ is the lifetime of lepton $\ell$, and the Yukawa couplings and the loop functions have been defined above.

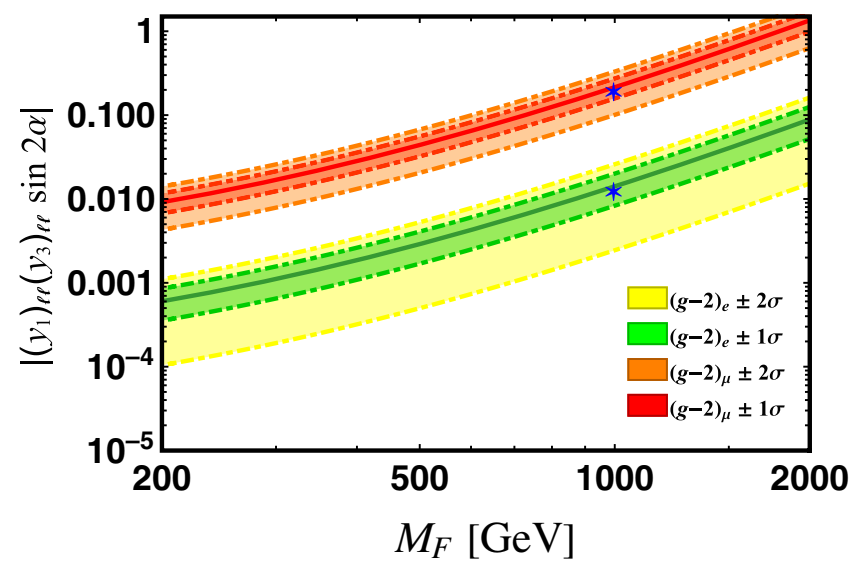

FIG. 3. The red (green) and orange (yellow) regions indicate the experimental $1 \sigma$ and $2 \sigma$ bands for the muon (electron) AMM $\Delta a_{\mu}$ $\left(\Delta a_{e}\right)$. The parameter space in Yukawa coupling vs vectorlike fermion mass plane consistent with both the electron and muon AMMs. Here, we choose the mass of scalars $S_{1}^{0}$ and $S_{2}^{0}$ to be 120 and $360 \mathrm{GeV}$, respectively. The blue star corresponds to the benchmark point given in Eqs. (3.21)-(3.24).

In Fig. 3, we show the parameter space in Yukawa coupling vs vectorlike lepton mass plane, which is consistent with the experimentally measured values of AMMs of the electron and muon. The red and orange regions correspond to the measured values of muon AMM within $1 \sigma$ and $2 \sigma$ allowed ranges, respectively, whereas green and yellow regions depict the parameter spaces consistent with the measured value of electron AMM within $1 \sigma$ and $2 \sigma$ allowed ranges, respectively. For illustration purpose, here we set the mass of the scalars $S_{1}^{0}\left(S_{2}^{0}\right)$ to be $120 \mathrm{GeV}$ $(360 \mathrm{GeV})$. The blue star in Fig. 3 indicates the benchmark point given in Eqs. (3.21)-(3.24).

\section{Neutrino mass}

The same vectorlike fermions play a major role in generating radiative neutrino mass, and the corresponding Feynman diagram is presented in Fig. 1. The loop is completed via the propagation of the singly charged scalars $S_{1,2}^{+}$, and we obtain the following expression for the neutrino mass matrix for model I:

$$
\begin{aligned}
\mathcal{M}_{i j}^{\nu}= & \frac{\sin 2 \gamma}{16 \pi^{2}} \sum_{\alpha=1}^{3}\left[\left(y_{1}\right)_{i \alpha}\left(y_{2}\right)_{j \alpha}+\left(y_{1}\right)_{j \alpha}\left(y_{2}\right)_{i \alpha}\right] M_{F_{\alpha}} \\
& \times\left[\frac{M_{S_{1}^{+}}^{2} \ln \frac{M_{S_{1}^{+}}^{2}}{M_{F_{\alpha}}^{2}}}{M_{S_{1}^{+}}^{2}-M_{F_{\alpha}}^{2}}-\frac{M_{S_{2}^{+}}^{2} \ln \frac{M_{2}^{2}}{M_{F_{\alpha}}^{2}}}{M_{S_{2}^{+}}^{2}-M_{F_{\alpha}}^{2}}\right] .
\end{aligned}
$$

Here, the mixing angle $\gamma$ between the singly charged physical particles is defined in Eq. (3.8). We stress here that for $\gamma=0$ the neutrino mass would vanish. This can be understood from the expressions in (3.2) and (3.8). To have 
nonzero Majorana mass for neutrinos, lepton number must be broken by two units. If we assign the lepton numbers $L\left[F_{L, R}, \phi_{1}, \phi_{2}, \eta\right]=\{+1,0,-2,0\}$ to the BSM fields, one can see that each of the terms in the Yukawa Lagrangian given in Eq. (3.1) respects lepton number conservation. This implies that the only term that breaks the lepton number by two units is the very last term in the scalar potential of Eq. (3.2). To be more specific, the simultaneous presence of the second, third, and fifth terms in Eq. (3.1) and the last term in Eq. (3.2) would imply that neutrino mass will be generated at loop level, once the heavy fields are integrated out. Vanishing $\gamma$ would correspond to vanishing $\lambda_{\phi_{1} \phi_{2}}^{\prime \prime}$, and thus no quartic scalar coupling of $\phi_{1}$ with $\phi_{2}$ and a SM Higgs pair. This in turn would correspond to the absence of the neutrino mass diagram in Fig. 1.

Note that to reproduce correct lepton mixing, one must have a nontrivial structure for the Yukawa coupling matrix $y_{2}$, since $y_{1}$ is taken to be diagonal. This, however, does not conflict with lepton flavor violating $\ell \rightarrow \ell^{\prime} \gamma$ processes mediated by the doubly charged scalars, since $\left(y_{2}\right)_{i j} \sim 10^{-5}$ in order to generate the correct neutrino mass scale. In the next subsection, we provide a realistic fit to neutrino mass spectrum. We would like to point out that a similar model for neutrino mass generation was considered in Ref. [73]; however, no such connections to lepton AMMs were made.

TABLE III. Current experimental values of the neutrino observables with their corresponding $1 \sigma$ uncertainties taken from Ref. [74].

\begin{tabular}{lccc}
\hline \hline Parameter & Best fit $\pm 1 \sigma$ & Parameter & Best fit $\pm 1 \sigma$ \\
\hline$\Delta m_{21}^{2}\left(10^{-5} \mathrm{eV}^{2}\right)$ & $7.50_{-0.20}^{+0.22}$ & $\sin ^{2} \theta_{12}$ & $0.318 \pm 0.016$ \\
$\Delta m_{31}^{2}\left(10^{-3} \mathrm{eV}^{2}\right)$ & $2.56_{-0.04}^{+0.03}$ & $\sin ^{2} \theta_{23}$ & $0.566_{-0.022}^{+0.016}$ \\
$\delta_{C P}$ & $1.20_{-0.14}^{+0.23} \pi$ & $\sin ^{2} \theta_{13}$ & $0.02225_{-0.00078}^{+0.00055}$ \\
\hline \hline
\end{tabular}

\section{Combined fit to data}

To demonstrate the viability of our proposed framework, here we present a combined fit to reproduce the experimental results. The expressions for $(g-2)_{e}$ and $(g-2)_{\mu}$ are given in Eq. (3.9), and their corresponding measured values can be found in Eqs. (1.1) and (1.2). Furthermore, from the neutrino mass formula Eq. (3.18), one needs to successfully incorporate two mass-squared differences, three mixing angles, and one Dirac $C P$ phase. The associated measured values in the experiments are summarized in Table III. The neutrino mass matrix can be parametrized as follows,

$$
\mathcal{M}_{\nu}=U_{\mathrm{PMNS}} \operatorname{diag}\left\{m_{1}, m_{2}, m_{3}\right\} U_{\mathrm{PMNS}}^{T},
$$

$$
U_{\mathrm{PMNS}}=\left(\begin{array}{ccc}
c_{12} c_{13} & s_{12} c_{13} & s_{13} e^{-i \delta} \\
-s_{12} c_{23}-c_{12} s_{23} s_{13} e^{i \delta} & c_{12} c_{23}-s_{12} s_{23} s_{13} e^{i \delta} & s_{23} c_{13} \\
s_{12} s_{23}-c_{12} c_{23} s_{13} e^{i \delta} & -c_{12} s_{23}-s_{12} c_{23} s_{13} e^{i \delta} & c_{23} c_{13}
\end{array}\right)\left(\begin{array}{ccc}
1 & 0 & 0 \\
0 & e^{i \frac{\alpha_{21}}{2}} & 0 \\
0 & 0 & e^{i \frac{\alpha_{31}}{2}}
\end{array}\right),
$$

where $m_{i}$ are real eigenvalues and we have defined $c_{i j}=\cos \theta_{i j}, s_{i j}=\sin \theta_{i j}$. In the PMNS mixing matrix, there exist three physical phases, one Dirac phase $\delta \equiv \delta_{C P}$ and two Majorana phases $\alpha_{21,31}$, where we have used the particle data group parametrization. In this work, we assume a normal ordering for neutrino masses that corresponds to $m_{1}<m_{2}<m_{3}$, which still is favored by oscillation data $[74,75]$.

With all these in hand, we can now perform a combined fit to the data. Since such a combined fit involves several parameters, to make life simple, we first fix the masses of the relevant BSM states, which corresponds to the set $\left\{M_{F_{\alpha}}, M_{S_{1}^{0}}, M_{S_{2}^{0}}, M_{S_{1}^{+}}, M_{S_{2}^{+}}\right\}$. Furthermore, we also fix the mixing angle $\alpha$ between the two mixed $C P$-even states. Then, we are left with one quartic coupling $\lambda_{\phi_{1} \phi_{2}}^{\prime \prime}$ and three Yukawa coupling matrices $y_{1,2,3}$. Following the above discussions, this corresponds to seven real and nine complex parameters to fit neutrino observables, the muon, and the electron AMMs. This is a multidimensional problem that involves several observables. To tackle this nondelete-linear problem, we optimize a $\chi^{2}$-function, which is defined as $\chi^{2}=\sum_{i} P_{i}^{2}$; here, the sum is taken over all the above-mentioned observables. Here, $P_{i}=$ $\left(T_{i}-O_{i}\right) / E_{i}$ is the pull for the observable $i$, and $T_{i}, O_{i}$, $E_{i}$ are the associated theory prediction, experimental central value, and $1 \sigma$ uncertainty of the measurement, respectively. It should be pointed out that, due to larger number of parameters compared to the number of observables, the $\chi^{2}$-function in the multidimensional parameter space is somewhat flat; hence, instead of searching for the global minimum, we are only interested in a sample local minimum. Following the aforementioned discussion, we perform a combined numerical analysis and provide a benchmark point in the following. For this benchmark point, the minimum corresponds to $\chi^{2}=0.3$, which is certainly an excellent fit to the data.

$$
\begin{aligned}
& M_{F_{\alpha}}=1 \mathrm{TeV} ; \quad M_{S_{1}^{0}}=0.12 \mathrm{TeV}, \quad M_{S_{2}^{0}}=3 M_{S_{1}^{0}} ; \\
& M_{S_{1}^{+}}=0.46 \mathrm{TeV} ; \quad M_{S_{2}^{+}}=3 M_{S_{1}^{+}},
\end{aligned}
$$




$$
\begin{gathered}
\sin \alpha=0.1, \lambda_{\phi_{1} \phi_{2}}^{\prime \prime}=8.325 \times 10^{-6}, \\
y_{1}=\left(\begin{array}{ccc}
0.3662 & 0 & 0 \\
0 & -1.0141 & 0 \\
0 & 0 & -0.43913
\end{array}\right), \quad y_{3}=\left(\begin{array}{ccc}
-0.19428 & 0 & 0 \\
0 & -1.07051 & 0 \\
0 & 0 & 0.14602
\end{array}\right), \\
y_{2}=10^{-5}\left(\begin{array}{ccc}
-0.10116+0.07932 i & -1.01099+0.62701 i & -0.49019-0.30200 i \\
-0.03471-0.04519 i & 0.31733-0.69407 i & 0.98401+0.8099 i \\
0.53005-0.9659 i & -0.05210+0.67329 i & -1.00145+0.82294
\end{array}\right)
\end{gathered}
$$

Yukawa couplings of order $y_{2} \sim 10^{-5}$ automatically satisfy all experimental constraints, including cLFV processes. The values of the theory parameters corresponding to this benchmark point successfully reproduce all the observables both in the neutrino sector as well as AMMs of the electron and the muon; we list the predictions in Table IV. Since an explanation of the lepton AMMs demands Yukawa couplings of order unity [as can be seen from Eq. (3.23)] and the same Yukawa couplings enter in neutrino mass generation, it can be easily understood that $y_{2} \lambda_{\phi_{1} \phi_{2}}^{\prime \prime} \sim 10^{-10}$ [instead of $y_{2}^{2} \sim 10^{-10}$ as in Eq. (2.1)] must be satisfied to reproduce the correct neutrino mass scale. Regarding the smallness of $\lambda_{\phi_{1} \phi_{2}}^{\prime \prime}$, we recall that it is the coefficient of the quartic coupling $\left(H \epsilon \phi_{1}\right)\left(\phi_{2}^{\dagger} H\right)$ responsible for mixing

TABLE IV. Fit values of some of the observables for our benchmark points given in Eqs. (3.21)-(3.24). Here, $m_{\cos }=$ $\sum_{i} m_{i}, m_{\beta}=\sqrt{\sum_{i}\left|U_{e i}\right|^{2} m_{i}^{2}}$ is the effective mass parameter for beta decay, and $m_{\beta \beta}=\left|\sum_{i} U_{e i}^{2} m_{i}\right|$ is the effective mass parameter for neutrinoless double beta decay.

\begin{tabular}{lc}
\hline \hline Quantity & Fit value \\
\hline$\Delta a_{e}$ & $-8.696 \times 10^{-13}$ \\
$\Delta a_{\mu}$ & $2.744 \times 10^{-9}$ \\
$\Delta m_{21}^{2}\left(10^{-5} \mathrm{eV}^{2}\right)$ & 7.525 \\
$\Delta m_{31}^{2}\left(10^{-3} \mathrm{eV}^{2}\right)$ & 2.552 \\
$\sin ^{2} \theta_{12}$ & 0.3171 \\
$\sin ^{2} \theta_{23}$ & 0.5638 \\
$\sin ^{2} \theta_{13}$ & 0.02216 \\
$\delta_{C P}$ & $223.8^{\circ}$ \\
\hline \hline Quantity & Fit value \\
\hline$m_{1}(\mathrm{eV})$ & 0.00812 \\
$m_{2}(\mathrm{eV})$ & 0.01188 \\
$m_{3}(\mathrm{eV})$ & 0.05117 \\
$m_{\mathrm{cos}}(\mathrm{eV})$ & 0.07118 \\
$m_{\beta}(\mathrm{eV})$ & 0.01207 \\
$m_{\beta \beta}(\mathrm{eV})$ & 0.00167 \\
$\alpha_{21}$ & $188.8^{\circ}$ \\
$\alpha_{31}$ & $311.9^{\circ}$ \\
\hline \hline
\end{tabular}

the two singly charged states, as defined in Eq. (3.8). In the limit of $\lambda_{\phi_{1} \phi_{2}}^{\prime \prime} \rightarrow 0$, neutrino masses are zero because the theory regains the accidental lepton number conservation of the SM. The chosen DM $\left(S_{1}^{0}\right)$ mass of $120 \mathrm{GeV}$ and the associated mixing angle $\sin \alpha$ for this benchmark point will be shown to be consistent with both DM detection bounds as well as DM relic abundance as detailed in the next section. It should be pointed out that in the DM analysis more parameters such as the Higgs portal coupling and lepton coupling portal play a role and are not fixed by the fit performed above. Moreover, the mass of the doubly charged scalar is not determined from the fit, which for simplicity we choose to be degenerate in mass with its singly charged partner to be consistent with $T$ parameter constraints. However, a splitting of order $\mathcal{O}(100) \mathrm{GeV}$ is still allowed [76].

\section{E. Dark matter phenomenology}

In this subsection, we analyze the DM phenomenology in model I, where lepton anomalous magnetic moments, neutrino masses, and mixings are successfully generated. As aforementioned, in this model, the presence of a discrete symmetry $\mathcal{Z}_{2}$ stabilizes the DM particle. The newly introduced scalars $\left(\phi_{1}, \phi_{2}\right.$, and $\left.\eta\right)$ and vectorlike leptons $F_{L, R}$ are odd under this discrete symmetry, whereas the SM particles are even. The lightest neutral particle among the new ones qualifies as a DM candidate. In our setup for model I, the dark matter candidate will be an admixture of neutral components of the doublet $\phi_{1}$ and the singlet $\eta$. As one can see from Eq. (3.9), one needs to introduce mixing between these two fields to successfully address electron and muon $g-2$ anomalies. Hence, the dark matter can be neither pure singlet type [77-89] nor pure inert doublet type [4,90-97]. Rather, it will be singlet-doublet scalar dark matter [98]. While scalar singlet dark matter is tightly constrained from DM direct detection experiments [86,88,99-101], inclusion of mixing with an additional doublet can introduce new additional interactions producing the right amount of relic density, which can potentially allow for evasion of direct detection bounds [99-101] for a large region of parameter space. 


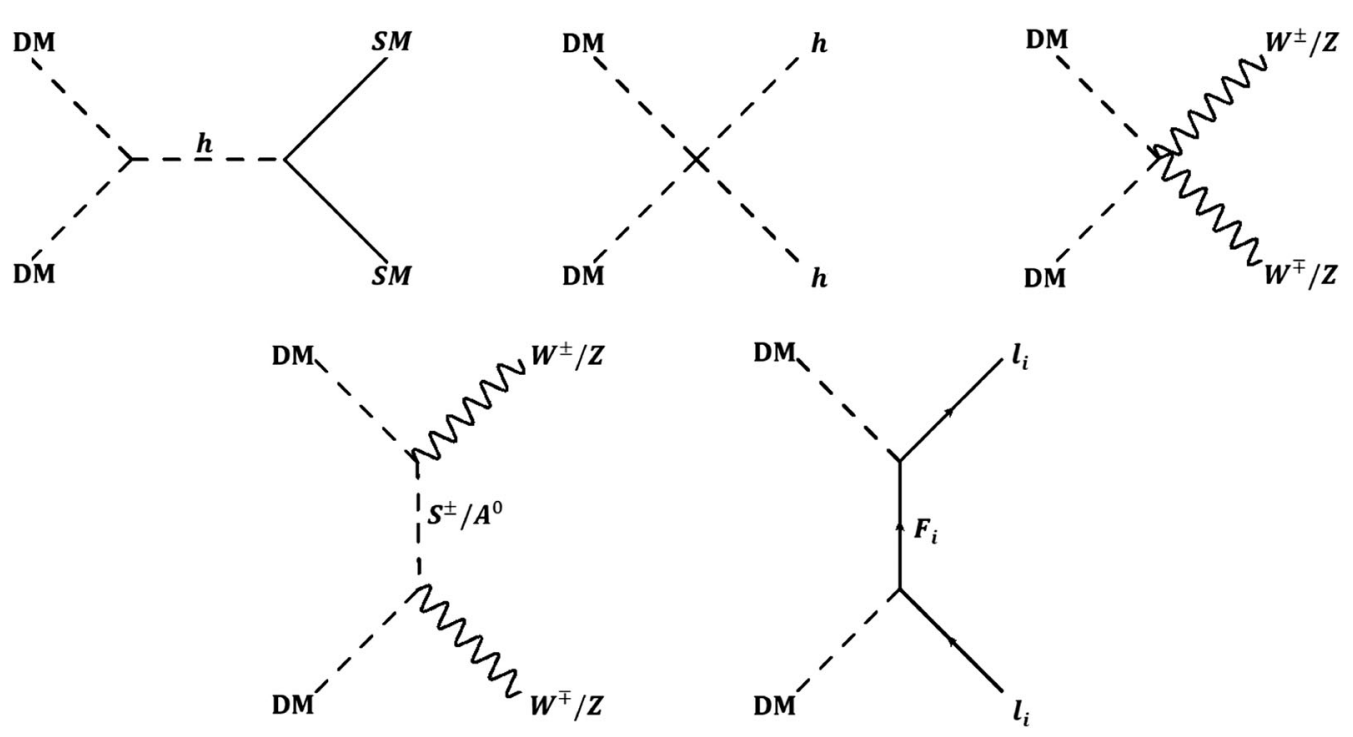

FIG. 4. Relevant Feynman diagrams that contribute to the annihilation of the DM.

The dominant processes that contribute to the annihilation of the DM particle are shown in Fig. 4. In our case, the DM can annihilate to SM particles through $s$-channel Higgs-mediated processes (Higgs portal). These Higssportal processes can be particularly important when the DM mass is close to half of the Higgs boson mass. Above this mass regime, the DM annihilation to gauge bosons (possible because it is partly a doublet) contributes dominantly to the annihilation processes. In this mass region, the DM annihilation through the $t$-channel exchange is usually smaller than the contribution from the four-point vertex shown in Fig. 4. Finally, the presence of the vectorlike leptons opens up new annihilation modes for DM via the $t$-channel processes (lepton portal) as shown in Fig. 4. In the low mass region, these leptonic portal processes become significant in addition to the Higgs-portal processes. For the DM analysis, we will denote the lepton portal coupling by $\lambda_{\mathrm{LP}}$. Since the DM is identified to be the state $S_{1}^{0}$, its lepton portal couplings with $F_{L_{j}}\left(F_{R_{j}}\right)$ is $\sqrt{2} Y_{L, 1}^{\ell j}\left(\sqrt{2} Y_{R, 1}^{\ell j}\right)$, which can be read off from Eqs. (3.12) and (3.13). On the other hand, $\lambda_{\mathrm{HP}}$ represents the Higgs portal coupling, which is defined by $\lambda_{\mathrm{HP}}=\left(\lambda_{H \phi_{1}}+\lambda_{H \phi_{1}}^{\prime}+2 \lambda_{H \phi_{1}}^{\prime \prime}\right) \sin ^{2} \alpha+2 \lambda_{H \eta} \cos ^{2} \alpha$. That is, our DM particle $S_{1}^{0}$ couples via

$$
\mathcal{L}=\frac{\lambda_{\mathrm{HP}}}{2}\left(S_{1}^{0}\right)^{2} H^{\dagger} H+\frac{\lambda_{\mathrm{LP}}}{\sqrt{2}} S_{1}^{0} \bar{e}_{L, R} F_{R, L}
$$

Let us quantify the DM phenomenology further. For our DM analysis, we have inserted our model in micrOMEGAs $[102,103]$ and performed a scan over the parameter space to analyze relic abundance and direct detection constraints. For the rest of the analysis, we fix the vector-ike lepton mass to be $1 \mathrm{TeV}$ (to be consistent with the benchmark point given in the previous section). As mentioned in the above paragraph, in our case, the viable DM mass range which is consistent with the WMAP relic density constraint can be divided into three regions. In the low mass regime $\left(m_{\mathrm{DM}} \lesssim 55 \mathrm{GeV}\right)$, the main annihilation channel of DM is via the leptonic $t$-channel processes, mediated by the vectorlike leptons. Since the $s$-wave and $p$-wave contributions of this leptonic channels are helicitysuppressed $[104,105]$, the $d$-wave contribution becomes dominant for the case of DM annihilation into electron-positron and muon-antimuon pairs. For DM annihilation into tau leptons, the $s$-wave and $p$-wave contributions become dominant compared to the $d$-wave contribution [104]. In Fig. 5 (bottom), we analyze the DM relic density as a function of DM mass for various leptonic portal couplings $\left(\lambda_{\mathrm{LP}}\right)$. Here, we set the Higgs portal coupling $\lambda_{\mathrm{HP}}=10^{-3}$ and the mixing angle $\sin \alpha=0.07$ for illustration. For simplicity, we also choose the leptonic portal coupling to be same for all the three leptons. For illustrating this further, we have also scanned the parameter space in Higgs-portal coupling $\left(\lambda_{\mathrm{HP}}\right)$ vs DM mass $\left(m_{\mathrm{DM}}\right)$ plane consistent with the WMAP relic density constraint for different choices of leptonic portal coupling in Fig. 6 (left). In the intermediate mass region $\left(55 \mathrm{GeV} \lesssim m_{\mathrm{DM}} \lesssim 75 \mathrm{GeV}\right.$ ), the dominant contribution to the DM annihilation comes from the $s$-channel Higgs mediated process. In Fig. 5 (top right), we analyze the DM relic density as a function of DM mass for various Higgs-portal couplings $\left(\lambda_{\mathrm{HP}}\right)$. The mixing angle $\sin \alpha=0.3$ and leptonic portal coupling $\lambda_{\mathrm{LP}}=0.1$ are chosen for better illustration. In the high mass regime $\left(m_{\mathrm{DM}} \gtrsim 75 \mathrm{GeV}\right)$, the relic density of DM depends on the mixing angle. In this parameter space, the dominant contribution to the DM annihilation cross section comes from the weak gauge bosons channels. In Fig. 5 (top left), we show the effect of varying the mixing angle on the relic density of DM for a fixed value of $\lambda_{\mathrm{HP}}=10^{-3}$ and $\lambda_{\mathrm{LP}}=0.1$. As the mixing 

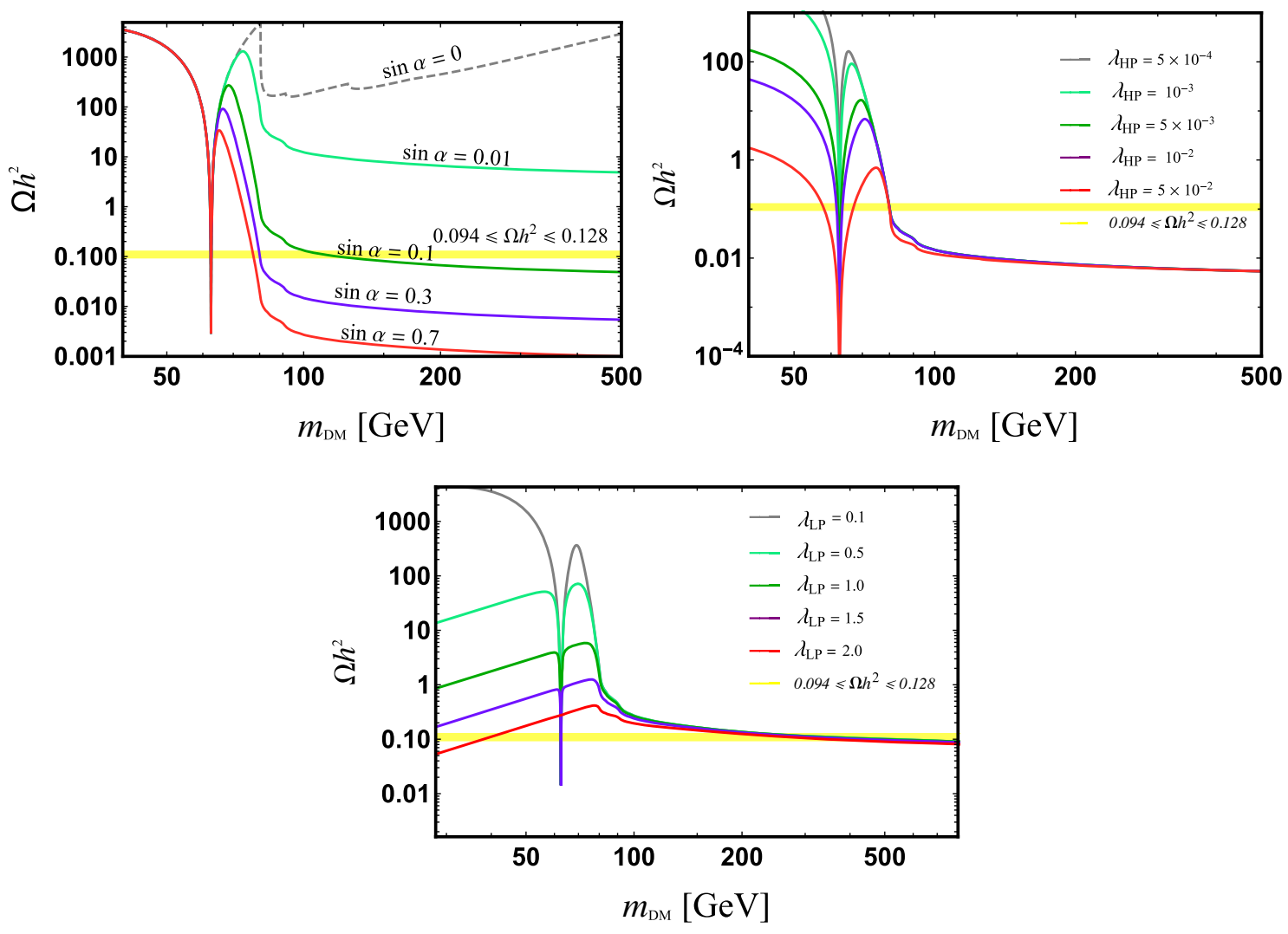

FIG. 5. DM relic density $\left(\Omega h^{2}\right)$ as a function of DM mass $\left(m_{\mathrm{DM}}\right)$. Top left: for different choices of mixing angle: $\sin \alpha=0.0$ (gray dotted), $\sin \alpha=0.01$ (light-green), $\sin \alpha=0.1$ (green), $\sin \alpha=0.3$ (violet), and $\sin \alpha=0.7$ (red). Here, we choose the Higgs-portal coupling $\lambda_{\mathrm{HP}}=10^{-3}$ and the leptonic portal coupling $\lambda_{\mathrm{LP}}=0.1$ for illustration. Top right: for various choices of the Higgs-portal coupling: $\lambda_{\mathrm{HP}}=5 \times 10^{-2}$ (red), $\lambda_{\mathrm{HP}}=10^{-2}$ (violet), $\lambda_{\mathrm{HP}}=5 \times 10^{-3}$ (green), $\lambda_{\mathrm{HP}}=10^{-3}$ (light green), and $\lambda_{\mathrm{HP}}=5 \times 10^{-4}$ (gray). The mixing angle $\sin \alpha=0.3$ and the leptonic portal coupling $\lambda_{\mathrm{LP}}=0.1$ are chosen for illustration. Bottom: for different choices of leptonic portal coupling: $\lambda_{\mathrm{LP}}=0.1$ (gray), $\lambda_{\mathrm{LP}}=0.5$ (light green), $\lambda_{\mathrm{LP}}=1.0$ (green), $\lambda_{\mathrm{LP}}=1.5$ (violet), and $\lambda_{\mathrm{LP}}=2.0$ (red). Here, we choose the Higgs-portal coupling $\lambda_{\mathrm{HP}}=10^{-3}$ and the mixing angle $\sin \alpha=0.07$ for illustration. The yellow band indicates the WMAP-observed relic density bound [106]. For all the panels, we set the vectorlike lepton mass to be $1 \mathrm{TeV}$.

angle increases, the annihilation cross section of DM into weak gauge bosons also increases. Due to this, the WMAP relic density constraint for DM can be satisfied for higher DM masses as well. For illustrating this, we also show the parameter space in Higgs-portal coupling $\left(\lambda_{\mathrm{HP}}\right)$ vs DM mass $\left(m_{\mathrm{DM}}\right)$ plane consistent with the WMAP relic density constraint for various choices of mixing angle in Fig. 6 (right).

In addition to the DM relic density study, we also consider the constraints from various DM direct detection experiments. In our model, the DM can interact with nuclei dominantly via $t$-channel Higgs boson exchange. The corresponding spin independent DM-nucleon scattering cross section is estimated in Refs. [78,88]. Using this, we recast the limits from LUX-2017 [100], PandaX-II [107], and XENON1T (2018) [99] experiments for our model, which are shown as brown, blue, and yellow regions, respectively, in Fig. 6. As one can see, we can satisfy all the present bounds from DM direct detection experiments for a large region of the parameter space.
Finally, in Fig. 7, we show the parameter space in the vectorlike lepton mass $\left(M_{F}\right)$ vs DM mass $\left(m_{\mathrm{DM}}\right)$ plane, which is consistent with the experimentally measured values of AMMs of the electron and muon, as well as the WMAP relic density constraint. The orange and yellow regions depict the parameter space which can address the electron and the muon $g-2$ anomalies, respectively. The pink, blue, and cyan shaded bands represent the parameter space consistent with the DM relic abundance $\left(0.094 \leq \Omega h^{2} \leq 0.128\right)$. For illustration, in left panel of Fig. 7, we set the Higgs-portal coupling $\lambda_{\mathrm{HP}}$ to be $10^{-3}$ and vary the mixing angle: $\sin \alpha=0.08$ (cyan), $\sin \alpha=0.1$ (blue), and $\sin \alpha=0.15$ (pink). On the other hand, in right panel of Fig. 7, the colored regions are shown for different choices of leptonic portal coupling that are consistent with the WMAP relic density constraint, $\lambda_{\mathrm{LP}}=0.146$ (cyan), $\lambda_{\mathrm{LP}}=1.5$ (blue), and $\lambda_{\mathrm{LP}}=2.0$ (pink), while fixing the mixing angle $\sin \alpha=0.1$ and the Higgs-portal coupling $\lambda_{\mathrm{HP}}=4 \times 10^{-4}$. For both the panels, we fix the product $\left(y_{1}\right)_{\ell \ell}\left(y_{3}\right)_{\ell \ell} \sin 2 \alpha$ to be same as the benchmark value 

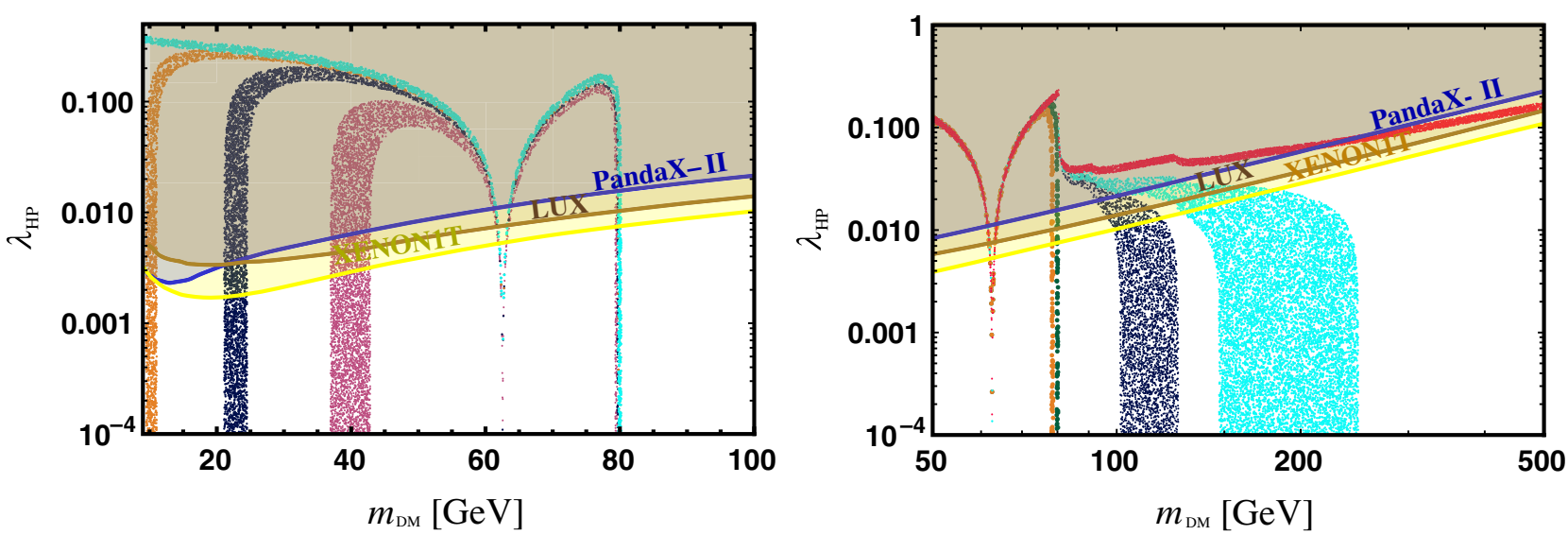

FIG. 6. The parameter space in Higgs-portal coupling $\left(\lambda_{\mathrm{HP}}\right)$ vs DM mass $\left(m_{\mathrm{DM}}\right)$ plane consistent with the WMAP relic density constraint. Left: regions are shown for different choices of leptonic portal coupling: $\lambda_{\mathrm{LP}}=0.5$ (cyan), $\lambda_{\mathrm{LP}}=1.0$ (orange), $\lambda_{\mathrm{LP}}=1.5$ (blue), and $\lambda_{\mathrm{LP}}=2.0$ (pink). Here, the mixing angle $\sin \alpha=0.3$ is kept fixed. Right: regions are shown for different choices of mixing angle: $\sin \alpha=0.01$ (red), $\sin \alpha=0.08$ (cyan), $\sin \alpha=0.1$ (blue), $\sin \alpha=0.3$ (green), and $\sin \alpha=0.5$ (orange). Here, we choose the leptonic portal coupling $\lambda_{\mathrm{LP}}=0.1$. The color shaded regions with solid boundary line denote the excluded parameter space by various current direct detection experiments: brown region from LUX-2017 [100], blue region from PandaX-II [107], and yellow region from XENON1T (2018) [99]. We set the vectorlike lepton mass to be $1 \mathrm{TeV}$.
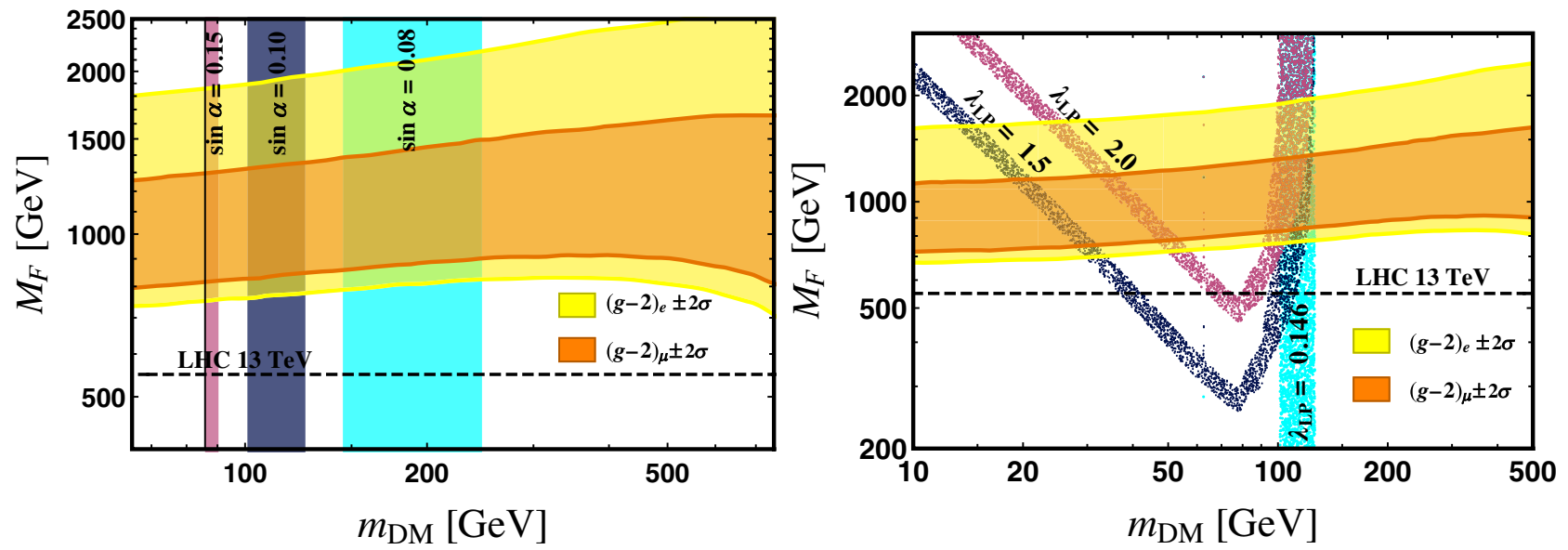

FIG. 7. The parameter space in vectorlike lepton mass $\left(M_{F}\right)$ vs DM mass $\left(m_{\mathrm{DM}}\right)$ plane consistent with both the electron and muon AMMs. The orange (yellow) region indicates the experimental $2 \sigma$ band for the muon (electron) AMM $\Delta a_{\mu}$ ( $\Delta a_{e}$ ). Left: the colored vertical bands represent the regions that are consistent with the WMAP relic density constraint for different choices of mixing angle: $\sin \alpha=0.08$ (cyan), $\sin \alpha=0.1$ (blue), and $\sin \alpha=0.15$ (pink). Here, we fix the Higgs-portal coupling $\lambda_{\mathrm{HP}}$ to be $10^{-3}$. Right: the colored regions are shown for different choices of leptonic portal coupling that are consistent with the WMAP relic density constraint: $\lambda_{\mathrm{LP}}=0.146$ (cyan), $\lambda_{\mathrm{LP}}=1.5$ (blue), and $\lambda_{\mathrm{LP}}=2.0$ (pink). Here, we fix the mixing angle sin $\alpha=0.1$ and the Higgs-portal coupling $\lambda_{\mathrm{HP}}=4 \times 10^{-4}$. For both the panels, we fix the product $\left(y_{1}\right)_{\ell \ell}\left(y_{3}\right)_{\ell \ell} \sin 2 \alpha$ to be same as the benchmark value given in Eqs. (3.21)-(3.24). The horizontal dashed line indicates the bound on the vectorlike lepton mass from the $13 \mathrm{TeV}$ LHC data [108].

given in Eqs. (3.21)-(3.24). The horizontal dashed line indicates the bound on the vectorlike lepton mass from the $13 \mathrm{TeV}$ LHC data [108]; cf. Sec. III F. As we can see from Fig. 7, there is a significant region of parameter space (intersection zones) which can accommodate the correct experimental values of $(g-2)_{e, \mu}$ as well as the DM relic abundance.

\section{F. Collider implications}

Here, we discuss the collider phenomenology associated with the dark matter in our model. Especially, the presence of doubly charged scalar $S^{ \pm \pm}$and the vectorlike leptons $F^{ \pm}$ can give rise to rich phenomenological implications at the LHC. Generically, DM is searched for at the LHC in mono- $X$ searches, e.g., in association with one or more 

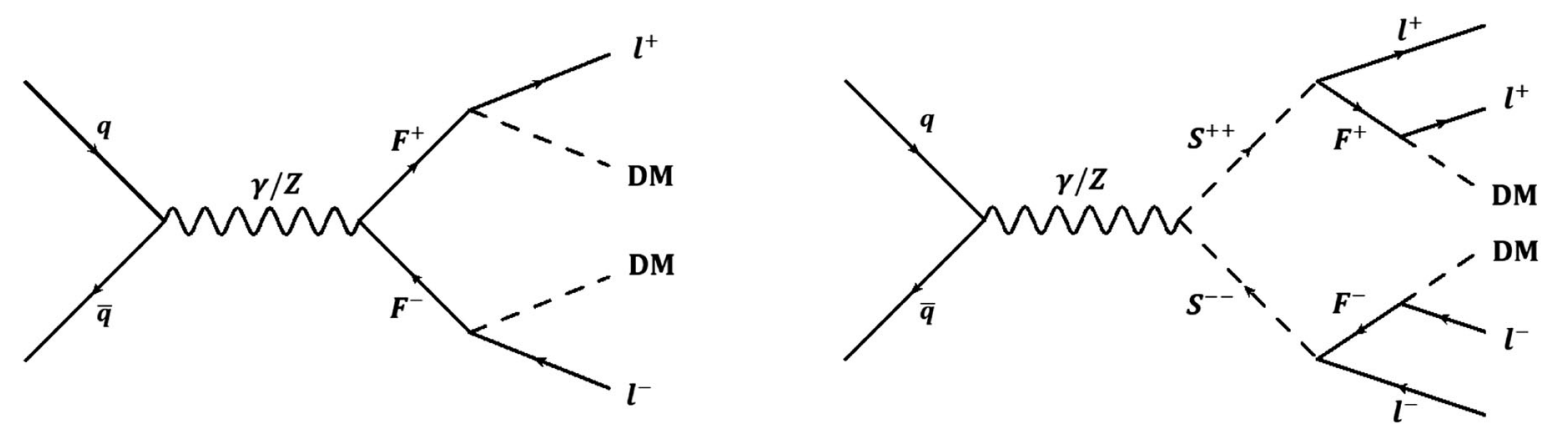

FIG. 8. Feynman diagrams for the collider signal of DM at the LHC.

additional SM particles, preferably a high momentum object (jet, photon, vector boson, etc.) radiated by the initial state quarks. Here, we want to highlight a few nonstandard collider aspects of DM which naturally arise in our framework. ${ }^{3}$ The relevant Feynman diagrams for this collider signal of DM are shown in Fig. 8. The charged vectorlike fermion $F^{ \pm}$, which is responsible for lepton anomalous magnetic moments, will be pair himproduced at the LHC via $s$-channel $Z / \gamma$ exchange, and it will further decay back to DM and SM charged leptons. This will lead to DM production in association with two charged leptons $\left(p p \rightarrow \ell^{+} \ell^{-}+\mathbb{E}_{T}\right)$ at the LHC. This process is somehow similar to the standard slepton searches [109-111]. If kinematically allowed, the DM can also be produced in association with same-sign dileptons from the decay of doubly charged scalar $S^{ \pm \pm}$as shown in right panel of the Fig. 8. The dominant production mechanism of the doubly charged scalar $S^{ \pm \pm}$at the LHC is the standard Drell-Yan process via $s$-channel $Z / \gamma$ exchange. It will further dominantly decay to $S^{ \pm \pm} \rightarrow F^{ \pm} \ell^{ \pm}$, and the vectorlike leptons $F^{ \pm}$decay dominantly to DM and SM charged leptons. This will lead to DM production in association with four charged leptons $\left(p p \rightarrow 2 \ell^{+} 2 \ell^{-}+\mathbb{E}_{T}\right)$ at the LHC. In addition to the pair production at the LHC, $S^{ \pm \pm}$can also be produced in association with the singly charged scalar $S_{2}^{ \pm}$via $W$ boson exchange. If kinematically allowed, $S^{ \pm \pm}$ can further decay into $S_{2}^{ \pm} W^{ \pm}$. Considering hadronic decay mode of $W$ boson, it will lead to a unique signature [73] at the LHC: $u \bar{d} \rightarrow S^{++} S_{2}^{-} \rightarrow\left(S_{2}^{+} W^{+}\right) S_{2}^{-} \rightarrow\left(l^{+} l^{-} j j+E /{ }_{T}\right)$. The prospects of this type of DM signal with multilepton signature were analyzed in details in Refs. [73,112]. On the other hand, if the $\mathcal{Z}_{2}$ odd charged scalars $S^{ \pm \pm}$and fermions $F^{ \pm}$are not kinematically allowed to decay to DM promptly, they will be long lived. In this case, the track originating from long-lived charged particles can disappear at a point inside the detector. There are dedicated searches for these stable charged particles at the LHC [108] using signatures

\footnotetext{
${ }^{3}$ Note that charged scalars in our benchmark point are chosen to be beyond $\mathrm{TeV}$, as are the vectorlike fermions. This implies that they are above current sensitivities, and we can keep the discussion largely qualitative.
}

of long time-of-flight measurements and anomalously high energy deposits in the silicon tracker. Nonobservation of any signal imposes severe constraints on these stable charged particles. Using the $13 \mathrm{TeV}$ LHC data [108], we find that the mass of a (long-lived) charged vectorlike fermion $F^{ \pm}$is constrained up to $550 \mathrm{GeV}$, whereas the mass limit on (long-lived) doubly charged scalar $S^{ \pm \pm}$is $660 \mathrm{GeV}$. Recently, displaced vertex and disappearing track signature for long-lived singly charged lepton were analyzed; see Refs. [113,114]. Also, the prospect of discovery of long-lived doubly charged scalars was analyzed [115]. Thus, this model predicts several unique signals like displaced vertex signature, disappearing tracks at the collider, and nonstandard DM signals with multilepton signature. All these signals have unique discovery prospects which can be tested in the upcoming run of the LHC or other colliders. ${ }^{4}$ The investigation of these collider signals is beyond the scope of this article and shall be presented in a future work.

\section{CONCLUSIONS}

In this work, we have proposed a class of models that intercorrelates and offers a simultaneous explanation of neutrino mass, dark matter, the long-standing puzzle of the muon anomalous magnetic moment, and the recently observed tension in the electron anomalous magnetic moment. In each of these models, the Standard Model is extended with a vectorlike fermion and a set of scalars, which are odd under an added $\mathcal{Z}_{2}$ symmetry. A common set of these BSM states runs through the loops and generates neutrino mass as well as lepton AMMs at one-loop order. If the vectorlike fermions are around the $\mathrm{TeV}$ scale, they provide large chirality enhanced contributions required to resolve the lepton AMMs. Different signs for the muon and the electron anomalous magnetic moments are arranged easily because different sets of Yukawa couplings are involved. The lightest of the neutral members of our

\footnotetext{
${ }^{4}$ For example, a future muon collider in the $\mathcal{O}(1)-\mathcal{O}(10) \mathrm{TeV}$ scale has the great potential to probe the class of models discussed in this work. For such relevant collider study, see Ref. [116].
} 
new multiplets, either fermionic or bosonic in nature, plays the role of the dark matter, which is stabilized by the unbroken $\mathcal{Z}_{2}$ symmetry. Models belonging to this class are simple in their constructions and provide a framework to unify a number of various seemly uncorrelated issues that cannot be solved with the Standard Model. After a generic discussion, we focused on a particular model and performed a detailed analysis that includes a fit to neutrino oscillation parameters as well as electron and muon
AMMs, followed by a discussion of DM and collider phenomenology.

\section{ACKNOWLEDGMENTS}

The work of V.P.K. was in part supported by U.S. Department of Energy Grant No. DE-SC 0016013. W. R. is supported by the DFG with GrantNo. RO 2516/7-1 in the Heisenberg program.
[1] G. Bertone, D. Hooper, and J. Silk, Particle dark matter: Evidence, candidates and constraints, Phys. Rep. 405, 279 (2005).

[2] Y. Cai, J. Herrero-Garcia, M. A. Schmidt, A. Vicente, and R. R. Volkas, From the trees to the forest: A review of radiative neutrino mass models, Front. Phys. 5, 63 (2017).

[3] K. S. Babu, P. S. B. Dev, S. Jana, and A. Thapa, Nonstandard interactions in radiative neutrino mass models, J. High Energy Phys. 03 (2020) 006.

[4] E. Ma, Verifiable radiative seesaw mechanism of neutrino mass and dark matter, Phys. Rev. D 73, 077301 (2006).

[5] D. Restrepo, O. Zapata, and C. E. Yaguna, Models with radiative neutrino masses and viable dark matter candidates, J. High Energy Phys. 11 (2013) 011.

[6] S. Jana, P. Vishnu, and S. Saad, Minimal realizations of Dirac neutrino mass from generic one-loop and two-loop topologies at $d=5$, J. Cosmol. Astropart. Phys. 04 (2020) 018.

[7] Y. Farzan, S. Pascoli, and M. A. Schmidt, Recipes and ingredients for neutrino mass at loop level, J. High Energy Phys. 03 (2013) 107.

[8] C. Simoes and D. Wegman, Radiative two-loop neutrino masses with dark matter, J. High Energy Phys. 04 (2017) 148.

[9] S. C. Chuliá, R. Cepedello, E. Peinado, and R. Srivastava, Systematic classification of two loop $d=4$ Dirac neutrino mass models and the Diracness-dark matter stability connection, J. High Energy Phys. 10 (2019) 093.

[10] S. Jana, P. K. Vishnu, and S. Saad, Minimal dirac neutrino mass models from $U(1)_{\mathrm{R}}$ gauge symmetry and left-right asymmetry at colliders, Eur. Phys. J. C 79, 916 (2019).

[11] M. Davier, A. Hoecker, B. Malaescu, and Z. Zhang, Reevaluation of the hadronic contributions to the Muon g-2 and to alpha(MZ), Eur. Phys. J. C 71, 1515 (2011).

[12] M. Davier, A. Hoecker, B. Malaescu, and Z. Zhang, Reevaluation of the hadronic vacuum polarisation contributions to the standard model predictions of the muon $g-2$ and $\alpha\left(m_{Z}^{2}\right)$ using newest hadronic cross-section data, Eur. Phys. J. C 77, 827 (2017).

[13] M. Davier, A. Hoecker, B. Malaescu, and Z. Zhang, A new evaluation of the hadronic vacuum polarisation contributions to the muon anomalous magnetic moment and to $\boldsymbol{\alpha}\left(\mathbf{m}_{\mathbf{Z}}^{2}\right)$, Eur. Phys. J. C 80, 241 (2020).
[14] T. Aoyama et al., The anomalous magnetic moment of the muon in the standard model, arXiv:2006.04822.

[15] G. Bennett et al. (Muon g-2 Collaboration), Final report of the Muon E821 anomalous magnetic moment measurement at BNL, Phys. Rev. D 73, 072003 (2006).

[16] R. H. Parker, C. Yu, W. Zhong, B. Estey, and H. Mueller, Measurement of the fine-structure constant as a test of the standard model, Science 360, 191 (2018).

[17] T. Aoyama, T. Kinoshita, and M. Nio, Revised and improved value of the QED tenth-order electron anomalous magnetic moment, Phys. Rev. D 97, 036001 (2018).

[18] G. Giudice, P. Paradisi, and M. Passera, Testing new physics with the electron g-2, J. High Energy Phys. 11 (2012) 113.

[19] H. Davoudiasl and W. J. Marciano, Tale of two anomalies, Phys. Rev. D 98, 075011 (2018).

[20] A. Crivellin, M. Hoferichter, and P. Schmidt-Wellenburg, Combined explanations of $(g-2)_{\mu, e}$ and implications for a large muon EDM, Phys. Rev. D 98, 113002 (2018).

[21] J. Liu, C. E. M. Wagner, and X.-P. Wang, A light complex scalar for the electron and muon anomalous magnetic moments, J. High Energy Phys. 03 (2019) 008.

[22] B. Dutta and Y. Mimura, Electron $g-2$ with flavor violation in MSSM, Phys. Lett. B 790, 563 (2019).

[23] X.-F. Han, T. Li, L. Wang, and Y. Zhang, Simple interpretations of lepton anomalies in the lepton-specific inert two-Higgs-doublet model, Phys. Rev. D 99, 095034 (2019).

[24] A. Crivellin and M. Hoferichter, Combined explanations of $(g-2)_{\mu},(g-2)_{e}$ and implications for a large muon EDM, in 33rd Rencontres de Physique de La Vallée d'Aoste (LaThuile 2019) La Thuile, Aosta, Italy (2019).

[25] M. Endo and W. Yin, Explaining electron and muon $g-2$ anomaly in SUSY without lepton-flavor mixings, J. High Energy Phys. 08 (2019) 122.

[26] M. Abdullah, B. Dutta, S. Ghosh, and T. Li, $(g-2)_{\mu, e}$ and the ANITA anomalous events in a three-loop neutrino mass model, Phys. Rev. D 100, 115006 (2019).

[27] M. Bauer, M. Neubert, S. Renner, M. Schnubel, and A. Thamm, Axion-like particles, lepton-flavor violation and a new explanation of $a_{\mu}$ and $a_{e}$, Phys. Rev. Lett. 124, 211803 (2020). 
[28] M. Badziak and K. Sakurai, Explanation of electron and muon $g-2$ anomalies in the MSSM, J. High Energy Phys. 10 (2019) 024.

[29] G. Hiller, C. Hormigos-Feliu, D. F. Litim, and T. Steudtner, arXiv:1910.14062 [Phys. Rev. D (to be published)].

[30] A. E. Cárcamo Hernández, S. F. King, H. Lee, and S. J. Rowley, Is it possible to explain the muon and electron $g-2$ in a $Z^{\prime}$ model?, Phys. Rev. D 101, 115016 (2020).

[31] C. Cornella, P. Paradisi, and O. Sumensari, Hunting for ALPs with lepton flavor violation, J. High Energy Phys. 01 (2020) 158.

[32] M. Endo, S. Iguro, and T. Kitahara, Probing $e \mu$ flavorviolating ALP at Belle II, J. High Energy Phys. 06 (2020) 040.

[33] A. E. C. Hernández, Y. H. Velásquez, S. Kovalenko, H. N. Long, N. A. Pérez-Julve, and V. V. Vien, Fermion masses and mixings and $g-2$ anomalies in a low scale 3-3-1 model, arXiv:2002.07347.

[34] N. Haba, Y. Shimizu, and T. Yamada, Muon and electron $g-2$ and the origin of Fermion mass hierarchy, Prog. Theor. Exp. Phys. 2020, 093 B05 (2020).

[35] I. Bigaran and R. R. Volkas, Getting chirality right: Topphilic scalar leptoquark solution to the $(g-2)_{e, \mu}$ puzzle, arXiv:2002.12544 [Phys. Rev. D (to be published)].

[36] S. Jana, Vishnu P. K., and S. Saad, Resolving electron and muon $g-2$ within the 2HDM, Phys. Rev. D 101, 115037 (2020).

[37] L. Calibbi, M. López-Ibáñez, A. Melis, and O. Vives, Muon and electron $g-2$ and lepton masses in flavor models, J. High Energy Phys. 06 (2020) 087.

[38] C.-H. Chen and T. Nomura, Electron and muon $g-2$, radiative neutrino mass, and $\ell^{\prime} \rightarrow \ell \gamma$ in a $U(1)_{e-\mu}$ model, arXiv:2003.07638.

[39] J.-L. Yang, T.-F. Feng, and H.-B. Zhang, Electron and muon $(g-2)$ in the B-LSSM, J. Phys. G 47, 055004 (2020).

[40] C. Hati, J. Kriewald, J. Orloff, and A. Teixeira, Anomalies in ${ }^{8} \mathrm{Be}$ nuclear transitions and $(g-2)_{e, \mu}$ : Towards a minimal combined explanation, J. High Energy Phys. 07 (2020) 235.

[41] B. Dutta, S. Ghosh, and T. Li, Explaining $(g-2)_{\mu, e}$, KOTO anomaly and MinibooNE excess in an extended Higgs model with sterile neutrinos, Phys. Rev. D 102, 055017 (2020).

[42] F. J. Botella, F. Cornet-Gomez, and M. Nebot, Electron and muon $g-2$ anomalies in general flavour conserving two Higgs doublets models, Phys. Rev. D 102, 035023 (2020).

[43] K.-F. Chen, C.-W. Chiang, and K. Yagyu, An explanation for the muon and electron $g-2$ anomalies and dark matter, J. High Energy Phys. 09 (2020) 119.

[44] I. Dorsner, S. Fajfer, and S. Saad, $\mu \rightarrow e \gamma$ selecting scalar leptoquark solutions for the $(g-2)_{e, \mu}$ puzzles, Phys. Rev. D 102, 075007 (2020).

[45] C. Arbeláez, R. Cepedello, R. M. Fonseca, and M. Hirsch, $(g-2)$ anomalies and neutrino mass, Phys. Rev. D 102, 075005 (2020).

[46] C.-S. Chen and C.-H. Chou, Neutrino masses, muon g-2, dark matter, lithium problem, and leptogenesis at $\mathrm{TeV}$ scale, Phys. Lett. B 699, 68 (2011).
[47] P. Agrawal, Z. Chacko, and C. B. Verhaaren, Leptophilic dark matter and the anomalous magnetic moment of the muon, J. High Energy Phys. 08 (2014) 147.

[48] S. Baek, Dark matter and muon $(g-2)$ in local $U(1)_{L_{\mu}-L_{\tau}{ }^{-}}$ extended Ma model, Phys. Lett. B 756, 1 (2016).

[49] G. Bélanger, C. Delaunay, and S. Westhoff, A dark matter relic from muon anomalies, Phys. Rev. D 92, 055021 (2015).

[50] K. Kowalska and E. M. Sessolo, Expectations for the muon $g-2$ in simplified models with dark matter, J. High Energy Phys. 09 (2017) 112.

[51] L. Calibbi, R. Ziegler, and J. Zupan, Minimal models for dark matter and the muon $g-2$ anomaly, J. High Energy Phys. 07 (2018) 046.

[52] B. Barman, D. Borah, L. Mukherjee, and S. Nandi, Correlating the anomalous results in $b \rightarrow s$ decays with inert Higgs doublet dark matter and muon $(g-2)$, Phys. Rev. D 100, 115010 (2019).

[53] C.-H. Chen and T. Nomura, Influence of an inert charged Higgs boson on the muon $g-2$ and radiative neutrino masses in a scotogenic model, Phys. Rev. D 100, 015024 (2019).

[54] L. Calibbi, T. Li, Y. Li, and B. Zhu, Simple model for large $C P$ violation in charm decays, B-physics anomalies, muon $g-2$, and dark matter, arXiv:1912.02676.

[55] E. Ma and M. Raidal, Neutrino Mass, Muon Anomalous Magnetic Moment, and Lepton Flavor Nonconservation, Phys. Rev. Lett. 87, 011802 (2001); Erratum, Phys. Rev. Lett. 87, 159901 (2001).

[56] D. A. Dicus, H.-J. He, and J. N. Ng, Neutrino-Lepton Masses, Zee Scalars and Muon $g-2$, Phys. Rev. Lett. 87, 111803 (2001).

[57] K. Babu and J. Julio, Two-loop neutrino mass generation through leptoquarks, Nucl. Phys. B841, 130 (2010).

[58] T. Nomura, H. Okada, and Y. Orikasa, $S U(2)_{L}$ septet scalar linking to a radiative neutrino model, Phys. Rev. D 94, 055012 (2016).

[59] T. Nomura and H. Okada, An extended colored Zee-Babu model, Phys. Rev. D 94, 075021 (2016).

[60] S. Lee, T. Nomura, and H. Okada, Radiatively induced neutrino mass model with flavor dependent gauge symmetry, Nucl. Phys. B931, 179 (2018).

[61] C.-W. Chiang, H. Okada, and E. Senaha, Dark matter, muon $g-2$, electric dipole moments, and $Z \rightarrow \ell_{i}^{+} \ell_{j}^{-}$in a one-loop induced neutrino model, Phys. Rev. D 96, 015002 (2017).

[62] S. Saad, Combined explanations of $(g-2)_{\mu}, R_{D^{(*)}}, R_{K^{(*)}}$ anomalies in a two-loop radiative neutrino mass model, Phys. Rev. D 102, 015019 (2020).

[63] C. Carloni Calame, M. Passera, L. Trentadue, and G. Venanzoni, A new approach to evaluate the leading hadronic corrections to the muon $g-2$, Phys. Lett. B 746, 325 (2015).

[64] P. S. B. Dev, W. Rodejohann, X.-J. Xu, and Y. Zhang, MUonE sensitivity to new physics explanations of the muon anomalous magnetic moment, J. High Energy Phys. 05 (2020) 053.

[65] A. Masiero, P. Paradisi, and M. Passera, New physics at the MUonE experiment at CERN, arXiv:2002.05418 [Phys. Rev. D (to be published)]. 
[66] T. Toma and A. Vicente, Lepton flavor violation in the Scotogenic model, J. High Energy Phys. 01 (2014) 160.

[67] M. Lindner, M. Platscher, and F. S. Queiroz, A call for new physics: The muon anomalous magnetic moment and lepton flavor violation, Phys. Rep. 731, 1 (2018).

[68] K. Babu and C. N. Leung, Classification of effective neutrino mass operators, Nucl. Phys. B619, 667 (2001).

[69] A. de Gouvea and J. Jenkins, A survey of lepton number violation via effective operators, Phys. Rev. D 77, 013008 (2008).

[70] P. W. Angel, N. L. Rodd, and R. R. Volkas, Origin of neutrino masses at the LHC: $\Delta L=2$ effective operators and their ultraviolet completions, Phys. Rev. D 87, 073007 (2013).

[71] J. P. Leveille, The second order weak correction to (G-2) of the muon in arbitrary gauge models, Nucl. Phys. B137, 63 (1978).

[72] B. L. Roberts and W. J. Marciano, Lepton dipole moments, Adv. Ser. Dir. High Energy Phys. 20, 1 (2009).

[73] M. Aoki, S. Kanemura, and K. Yagyu, Doubly-charged scalar bosons from the doublet, Phys. Lett. B 702, 355 (2011); Erratum, Phys. Lett. B 706, 495 (2012).

[74] P. de Salas, D. Forero, S. Gariazzo, P. Martínez-Miravé, O. Mena, C. Ternes, M. Tórtola, and J. Valle, 2020 Global reassessment of the neutrino oscillation picture, arXiv: 2006.11237.

[75] I. Esteban, M. Gonzalez-Garcia, M. Maltoni, T. Schwetz, and A. Zhou, The fate of hints: updated global analysis of three-flavor neutrino oscillations, arXiv:2007.14792.

[76] S. Kanemura and K. Yagyu, Radiative corrections to electroweak parameters in the Higgs triplet model and implication with the recent Higgs boson searches, Phys. Rev. D 85, 115009 (2012).

[77] T. Steele, Z.-W. Wang, D. Contreras, and R. Mann, Viable Dark Matter via Radiative Symmetry Breaking in a Scalar Singlet Higgs Portal Extension of the Standard Model, Phys. Rev. Lett. 112, 171602 (2014).

[78] J. M. Cline, K. Kainulainen, P. Scott, and C. Weniger, Update on scalar singlet dark matter, Phys. Rev. D 88, 055025 (2013); Erratum, Phys. Rev. D 92, 039906 (2015).

[79] W.-L. Guo and Y.-L. Wu, The Real singlet scalar dark matter model, J. High Energy Phys. 10 (2010) 083.

[80] A. Bandyopadhyay, S. Chakraborty, A. Ghosal, and D. Majumdar, Constraining scalar singlet dark matter with CDMS, XENON and DAMA and prediction for direct detection rates, J. High Energy Phys. 11 (2010) 065.

[81] C. Burgess, M. Pospelov, and T. ter Veldhuis, The minimal model of nonbaryonic dark matter: A singlet scalar, Nucl. Phys. B619, 709 (2001).

[82] J. McDonald, Gauge singlet scalars as cold dark matter, Phys. Rev. D 50, 3637 (1994).

[83] C. E. Yaguna, The singlet scalar as FIMP dark matter, J. High Energy Phys. 08 (2011) 060.

[84] G. Belanger, K. Kannike, A. Pukhov, and M. Raidal, $Z_{3}$ scalar singlet dark matter, J. Cosmol. Astropart. Phys. 01 (2013) 022.

[85] A. Drozd, B. Grzadkowski, J. F. Gunion, and Y. Jiang, Extending two-Higgs-doublet models by a singlet scalar field-The case for dark matter, J. High Energy Phys. 11 (2014) 105.
[86] L. Feng, S. Profumo, and L. Ubaldi, Closing in on singlet scalar dark matter: LUX, invisible Higgs decays and gamma-ray lines, J. High Energy Phys. 03 (2015) 045.

[87] S. Bhattacharya, S. Jana, and S. Nandi, Neutrino masses and scalar singlet dark matter, Phys. Rev. D 95, 055003 (2017).

[88] S. Bhattacharya, P. Poulose, and P. Ghosh, Multipartite interacting scalar dark matter in the light of updated LUX data, J. Cosmol. Astropart. Phys. 04 (2017) 043.

[89] G. Arcadi, A. Djouadi, and M. Raidal, Dark matter through the Higgs portal, Phys. Rep. 842, 1 (2020).

[90] R. Barbieri, L. J. Hall, and V. S. Rychkov, Improved naturalness with a heavy Higgs: An alternative road to LHC physics, Phys. Rev. D 74, 015007 (2006).

[91] M. Gustafsson, E. Lundstrom, L. Bergstrom, and J. Edsjo, Significant Gamma Lines from Inert Higgs Dark Matter, Phys. Rev. Lett. 99, 041301 (2007).

[92] P. Agrawal, E. M. Dolle, and C. A. Krenke, Signals of inert doublet dark matter in neutrino telescopes, Phys. Rev. D 79, 015015 (2009).

[93] E. Nezri, M. H. Tytgat, and G. Vertongen, $e^{+}$and $\bar{p}$ from inert doublet model dark matter, J. Cosmol. Astropart. Phys. 04 (2009) 014.

[94] C. Arina, F.-S. Ling, and M. H. Tytgat, IDM and iDM or the inert doublet model and inelastic dark matter, J. Cosmol. Astropart. Phys. 10 (2009) 018.

[95] J.-O. Gong, H. M. Lee, and S. K. Kang, Inflation and dark matter in two Higgs doublet models, J. High Energy Phys. 04 (2012) 128.

[96] L. L. Honorez, E. Nezri, J. F. Oliver, and M. H. Tytgat, The inert doublet model: An archetype for dark matter, J. Cosmol. Astropart. Phys. 02 (2007) 028.

[97] A. Goudelis, B. Herrmann, and O. Stal, Dark matter in the inert doublet model after the discovery of a Higgs-like boson at the LHC, J. High Energy Phys. 09 (2013) 106.

[98] T. Cohen, J. Kearney, A. Pierce, and D. Tucker-Smith, Singlet-doublet dark matter, Phys. Rev. D 85, 075003 (2012).

[99] E. Aprile et al. (XENON Collaboration), Dark Matter Search Results from a One Ton-Year Exposure of XENON1T, Phys. Rev. Lett. 121, 111302 (2018).

[100] D. Akerib et al. (LUX Collaboration), Results from a Search for Dark Matter in the Complete LUX Exposure, Phys. Rev. Lett. 118, 021303 (2017).

[101] X. Cui et al. (PandaX-II Collaboration), Dark Matter Results From 54-Ton-Day Exposure of PandaX-II Experiment, Phys. Rev. Lett. 119, 181302 (2017).

[102] G. Bélanger, F. Boudjema, A. Pukhov, and A. Semenov, micromegAs4.1: Two dark matter candidates, Comput. Phys. Commun. 192, 322 (2015).

[103] G. Bélanger, F. Boudjema, A. Goudelis, A. Pukhov, and B. Zaldivar, micromegAs5.0: Freeze-in, Comput. Phys. Commun. 231, 173 (2018).

[104] A. Gaviria, R. Longas, and A. Rivera, Lepton dark matter portal in the inert Zee model, arXiv:2005.13687.

[105] A. Berlin, D. Hooper, and S. D. McDermott, Simplified dark matter models for the Galactic Center gamma-ray excess, Phys. Rev. D 89, 115022 (2014).

[106] G. Hinshaw, D. Larson, E. Komatsu, D. N. Spergel, C. L. Bennett, J. Dunkley, M. R. Nolta, M. Halpern, R. S. Hill, N. Odegard et al., Nine-year wilkinson microwave 
anisotropy probe (wmap) observations: Cosmological parameter results, Astrophys. J. Suppl. Ser. 208, 19 (2013).

[107] Q. Wang et al., Results of dark matter search using the full PandaX-II exposure, arXiv:2007.15469.

[108] V. Khachatryan et al. (CMS Collaboration), Search for long-lived charged particles in proton-proton collisions at $\sqrt{s}=13 \mathrm{TeV}$, Phys. Rev. D 94, 112004 (2016).

[109] A. M. Sirunyan et al. (CMS Collaboration), Search for supersymmetry in events with a $\tau$ lepton pair and missing transverse momentum in proton-proton collisions at $\sqrt{s}=13$ TeV, J. High Energy Phys. 11 (2018) 151.

[110] G. Aad et al. (ATLAS Collaboration), Search for the direct production of charginos, neutralinos and staus in final states with at least two hadronically decaying taus and missing transverse momentum in $p p$ collisions at $\sqrt{s}=$ $8 \mathrm{TeV}$ with the ATLAS detector, J. High Energy Phys. 10 (2014) 096.

[111] A. M. Sirunyan et al. (CMS Collaboration), Search for supersymmetric partners of electrons and muons in proton-proton collisions at $\sqrt{s}=13 \mathrm{TeV}$, Phys. Lett. B 790, 140 (2019).

[112] S. Chakraborti and R. Islam, Implications of dark sector mixing on leptophilic scalar dark matter, arXiv:2007 .13719.

[113] S. Jana, N. Okada, and D. Raut, Displaced vertex and disappearing track signatures in type-III Seesaw, arXiv: 1911.09037.

[114] B. Acharya, A. De Roeck, J. Ellis, D. Ghosh, R. Masełek, G. Panizzo, J. Pinfold, K. Sakurai, A. Shaa, and A. Wall, Prospects of searches for long-lived charged particles with MoEDAL, Eur. Phys. J. C 80, 572 (2020).

[115] P. B. Dev and Y. Zhang, Displaced vertex signatures of doubly charged scalars in the type-II seesaw and its left-right extensions, J. High Energy Phys. 10 (2018) 199.

[116] R. Capdevilla, D. Curtin, Y. Kahn, and G. Krnjaic, A guaranteed discovery at future muon colliders, arXiv: 2006.16277 . 